\title{
Thermomechanical processing of metallic glasses: extending the range of the glassy state
}

\author{
Yonghao Sun ${ }^{1}$, Amadeu Concustell ${ }^{2}$ and A. Lindsay Greer ${ }^{1,3}$ \\ ${ }^{1}$ Department of Materials Science \& Metallurgy, University of Cambridge, UK \\ ${ }^{2}$ Thermal Spray Centre (CPT), University of Barcelona, Spain \\ ${ }^{3}$ WPI Advanced Institute for Materials Research, Tohoku University, Sendai, Japan.
}

\begin{abstract}
For crystalline metals the science, technology, and application of thermomechanical processing are established, but this is not true for glasses. Metallic glasses - because they can be plastically deformed - offer a unique opportunity to study the effects of thermomechanical treatments on the structure and properties of glasses. Depending on the rate of cooling, various glassy states can form from a liquid. Slower cooling gives states of lower enthalpy and smaller volume; such states might also be reached by annealing, which induces structural 'relaxation'. A reduction in the degree of relaxation, or 'rejuvenation', is achievable through processes such as irradiation and mechanical deformation. In this Review, we explore the extent of relaxation and rejuvenation induced by thermomechanical processing (elastic and plastic deformation, including cold and hot working and cyclic loading). The issues that remain to be investigated and the prospects for further progress are discussed.
\end{abstract}

\section{Web summary}

Thermomechanical treatments — cold and hot deformation — are established for polycrystalline metals. Their use on metallic glasses to obtain relaxed and rejuvenated states has just begun to be explored, and holds promise for extending the range of available glassy states and gaining access to improved properties.

Metallic glasses are amorphous solids formed by cooling a metallic melt fast enough to avoid crystallization. First reported in $1960^{1}$, metallic glasses are now known to form at very many compositions ${ }^{2-4}$ and are the focus of a sustained research effort motivated by both fundamental and practical interest ${ }^{5-7}$. The kinetic nature of the transition from liquid to glass (BOX 1) ensures that there is a range of possible glassy states. In this Review, we discuss results that suggest the range of practically accessible states can be extended, and we consider the prospects for accessing desirable properties.

For conventional polycrystalline metals, the range of microstructures obtained upon casting from the liquid can be greatly extended by thermal and mechanical 
treatments. For conventional glasses (for example, oxides), however, mechanical treatments are almost unknown because these glasses are brittle and cannot be plastically deformed at room temperature, at least not in their bulk form. By contrast, metallic glasses can show considerable plasticity, which opens up the possibility of studying the effects of deformation on their structure and properties. Intriguingly, these include the effects of deformation within the elastic range, something hardly explored for conventional polycrystalline metals.

\section{Box 1 | The glassy state}

A glass is produced if crystallization is avoided when cooling a liquid to form a solid. It is easier to compare the phases and their properties - for example, volume, enthalpy and entropy - by restricting our consideration to a system in which all phases have the same composition. Below the melting temperature, $T_{\mathrm{m}}$, the thermodynamically stable state (that is, the crystalline state, blue line in the illustration) can be avoided if cooling is fast enough to prevent significant crystal nucleation and growth. The properties of the liquid (red line in the illustration) are more strongly dependent on temperature than those of the crystalline and glassy solid states (blue and purple lines respectively). The contraction upon cooling observed in the solid states arises from a reduction in anharmonic vibrations, decreasing the average bond length. This effect is present in liquids, but it is accompanied by a structural change. As the liquid is cooled, it becomes more densely packed (for metals, this can include an increase in the nearest-neighbour coordination number of the atoms). This denser liquid becomes more viscous, with an increased structural relaxation time. If this time exceeds a characteristic value set by the experimental cooling rate, structural evolution stops; this occurs at the $T_{\mathrm{g}}$. At a cooling rate of $20 \mathrm{~K} \mathrm{~min}^{-1}$, this glass transition is conventionally taken to occur at a liquid viscosity of $10^{12} \mathrm{~Pa}$ s. Below $T_{\mathrm{g}}$ the material is in the glassy state and has a structure that, ideally, no longer changes by further decreasing the temperature.

\section{Box 1 Figure near here}

The slower the liquid is cooled, the lower the glass-transition temperature ( $T_{\mathrm{ga}}$ in the illustration), and the glass that is formed has a lower enthalpy and is more dense (line $\mathrm{a}$ in the illustration). By contrast, faster cooling gives a less dense glass that is formed at a higher glass-transition temperature ( $T_{\mathrm{gb}}$, line $\mathrm{b}$ in the illustration). The formation of a glass is a kinetic phenomenon and $T_{\mathrm{g}}$ is not thermodynamically defined. If there is atomic mobility, a glass evolves to states of lower enthalpy and volume in a process called 'structural relaxation' or 'ageing'. The converse process is 
called 'rejuvenation' and, if restricted to thermal treatments, it is possible only by heating the glass back into the liquid state and quenching rapidly.

Metallic glasses lack the microstructural features associated with conventional alloys, such as grain and twin boundaries or multiphase dispersions. Their uniformity results, for example, in soft magnetic properties (lack of domain-wall pinning) and resistance to corrosion (lack of chemical segregation and local attack).

Early work on metallic glasses focused on rapidly quenched $\left(10^{5}-10^{6} \mathrm{~K} \mathrm{~s}^{-1}\right)$ ribbons, a few tens of micrometres thick, produced by melt-spinning ${ }^{8}$. Soft-magnetic Fe-based ribbons remain the basis of the main commercial applications of metallic glasses $^{5,7}$. In recent years, attention has turned to the development of multicomponent compositions allowing the formation of bulk metallic glasses at low cooling rates; these materials, which are available as fully glassy cylinders with diameters of up to several centimetres, are of interest primarily for their mechanical properties ${ }^{3,5,9}$. Metallic glasses have exceptionally high yield stresses compared to crystalline metallic materials because of the absence of crystallographic slip systems. They have the highest known yield strength of all metallic materials if expressed as a fraction of the ideal strength ${ }^{9}$.

The bulk moduli of metallic glasses are similar to those of their crystalline counterparts, whereas their shear moduli are significantly lower (by $\sim 30 \%$ ). In addition, the yield strain of metallic glasses under uniaxial loading $(\sim 2 \%)$ is much larger than that of typical crystalline alloys. In contrast to crystalline alloys, plastic deformation is accompanied by softening, and flow is sharply localized in shear bands, (that is thin planes within which the shearing is very intense). Under uniaxial tension, the formation of such bands leads to early failure; thus the ductility (the plasticity in tension) is effectively zero. However, the fracture toughness can be high, with values of up to $232 \mathrm{MPa} \mathrm{m}^{1 / 2}$ [REF. 10]. Upon annealing, metallic glasses relax to lowerenergy states that are often brittle. The reverse process, termed rejuvenation, brings the materials into higher-energy, less brittle states (BOX 1), and is therefore attractive.

There are many reasons for interest in a wider range of glassy states. Different glassy states can have a variety of characteristics and hence, possible applications. For example, rejuvenated states offer improvements in plasticity and in catalytic and tribological properties; they may also be of interest for rapid crystallization in applications based on phase switching ${ }^{11}$. Relaxed states exhibit high yield stress, hardness, low damping, low creep, high magnetic permeability, diffusion-barrier properties, greater chemical stability and high resistance to crystallization.

There has been much work, including some mechanical processing ${ }^{12}$, on composite materials based on bulk metallic glasses, in particular, to optimize their 
mechanical properties ${ }^{4}$. In this Review, we focus on monolithic bulk metallic glasses, but we note that heterogeneities within glassy phases can be exploited to improve properties.

\section{Mechanical processing of alloys}

Engineering alloys are typically ductile, and plastic deformation is used to shape them and to change their properties. One important phenomenon in the processing of engineering alloys is work-hardening. Plastic deformation of crystalline metals is mediated by dislocation glide, and generates more dislocations impeding the glide, providing work-hardening. Annealing a work-hardened sample results in relaxation toward lower energy (recovery), but the only way to substantially reduce the dislocation density is to recrystallize the sample. Recrystallization occurs above a critical temperature $T_{\mathrm{R}}$ (typically around $60 \%$ of the absolute melting temperature, $T_{\mathrm{m}}$ ); at this temperature a new microstructure is formed through nucleation of relatively dislocation-free grains, reducing the grain size. Processes such as rolling or wire-drawing are directional, and induce a preferred orientation (crystallographic texture) into the polycrystalline structure. After recrystallization there is often a strong, but different, texture. Cold-working of metals is performed below $T_{\mathrm{R}}$, and usually at room temperature; hot-working is carried out above $T_{\mathrm{R}}$, and in this case, because of ongoing recrystallization, work-hardening is avoided.

The possibility of inducing useful changes in the metal's structure and properties has motivated the development of thermomechanical processing of conventional alloys. Potentially complex sequences of heating, working and cooling, if applied to optimized compositions, can be used to tailor the properties of the resultant materials ${ }^{13}$. Thermomechanical processing, for example, can improve the microstructures of as-cast alloys by making them finer and more uniform. In glasses, thermomechanical processing is virtually unexplored, yet the plasticity of metallic glasses clearly offers opportunities for structural change and property optimization.

\section{Deformation in metallic glasses}

Elastic deformation. The elastic moduli of metallic glasses, which are low compared to those of their crystalline counterparts ${ }^{14}$, can be understood in terms of local atomic rearrangements, even in the nominally elastic regime ${ }^{15}$. At the atomic level, elastic strain in a glassy structure is non-affine ${ }^{16}$ (that is, inhomogeneous), because the atoms never occupy equivalent positions as they do in a crystal ${ }^{17}$. Even at very low macroscopic strain there can be local strains that are high enough to trigger 
rearrangements of topologically unstable atomic configurations ${ }^{18}$. Although similar rearrangements occur in the reverse sense upon unloading, contributing to an apparently elastic behaviour, the original atomic arrangements are not exactly restored $^{17}$, and there is a net structural change. Compared to crystalline metals, metallic glasses show unusually large anelastic (that is, time-dependent, but fully recoverable) strain ${ }^{17,19}$. Upon loading, local plasticity — rearrangement in soft spots - is observed within a comparatively rigid matrix and, upon unloading, the elastically deformed matrix pushes the soft spots back into (roughly) their original configurations. Up to $\sim 24 \%$ of recoverable deformation may be anelastic ${ }^{17,20}$. A glass with anelastic strain is anisotropic and displays a Bauschinger effect, meaning that it is easier to reverse a deformation than to continue deforming in the same direction $^{21}$.

\section{Figure 1 near here}

Plastic deformation. For a metallic glass and its corresponding liquid a schematic universal map ${ }^{22}$ (FIG. 1a) can be used to show contours of flow rate (specifically shear strain rate $\dot{\gamma}$ as a function of normalized shear stress $\tau$ and temperature. At temperatures much higher than the glass-transition temperature, $T_{\mathrm{g}}$, relaxation is fast and the structure of the liquid stays in equilibrium, not affected by shear. The flow of the liquid is Newtonian $(\dot{\gamma} \propto \tau)$ : at a given temperature the viscosity is constant (independent of $\dot{\gamma}$ ), and $\tau$ is independent of strain (FIG. 1b). Upon cooling, the viscosity increases rapidly (indicated in FIG. 1a by the increase of $\tau$ for a given $\dot{\gamma}$ ) as $T_{\mathrm{g}}$ is approached, and in the glassy state it continues to increase, albeit less strongly. Below $T_{\mathrm{g}}$, homogeneous flow at a given $\dot{\gamma}$ increases disorder in the glassy structure, reducing its viscosity, and decreasing $\tau$ (FIG. 1b). A non-equilibrium steady-state viscosity that depends on $\dot{\gamma}$ is established; the flow is therefore non-Newtonian. At higher $\tau$ and $\dot{\gamma}$ flow-induced disordering leads to an instability (red line in FIG. 1c) in which the flow is no longer homogeneous, but concentrated into one or more 'shear bands'. The onset of yield is observed, soon followed by catastrophic failure (FIG. 1b). The nature of the instability is such that the bands are very thin, 10-20 nm [REF. 23], and $\tau$ is nearly independent of $\dot{\gamma}$ (FIG. 1a).

Thus, metallic glasses show two types of plastic deformation (FIG. 1a) ${ }^{22}$. Under low stress, a homogeneous viscous flow is observed, in which each volume element of the glass behaves similarly. Below $T_{\mathrm{g}}$, this flow is slow and can be described as creep. Above $T_{\mathrm{g}}$, the flow can be rapid, which makes it possible to shape metallic glasses in a similar way to polymer and oxide glasses. Under high stress, over a broad temperature range that includes room temperature, the plastic flow is inhomogeneous, 
localized into shear bands. In this regime, the shear yield stress at room temperature scales with the shear modulus, so that the shear yield strain has an average value of $(2.67 \pm 0.02) \%$. This behaviour has been analysed in terms of the cooperative shear model originally developed for dislocation-free crystals ${ }^{24}$. The presence of many shear bands can result in substantial plasticity. Even so, shear banding is the aspect of mechanical behaviour of metallic glasses that mainly limits their wider application, because it leads to catastrophic failure in tension and to unattractive surface markings $^{23}$.

The mechanisms governing plastic flow in crystalline metals do not apply to metallic glasses. From studies of $2 \mathrm{D}$ bubble rafts, it has emerged that the relevant flow units in disordered systems are shear transformation zones ${ }^{25}$. These zones are small spheroidal regions in which local shear occurs in response to stress. Unlike dislocations in crystals, shear transformation zones cannot be imaged at the microscopic level, but their detection and that of their strain fields is possible in colloidal glasses ${ }^{26}$. As a result, colloidal glasses may be useful analogues for metallic glasses. The behaviour of shear transformation zones has been widely studied through atomistic simulations and associated modelling ${ }^{27-30}$. These zones show a distribution of sizes and activation energies, and their presence leads to redistribution of local stresses and to rearrangements that may lead to overall relaxation or rejuvenation. If the applied stress is sufficiently high, the behaviour of shear transformation zones becomes cooperative, and the result is an avalanche effect that is manifested as shear banding ${ }^{31}$.

\section{Figure 2 near here}

\section{The working of metallic glasses}

Cold-working. In this section, we discuss the room-temperature deformation of metallic glasses. Metallic glasses usually show no general plasticity in tension because of shear banding, but substantial plastic flow can be achieved by processing them under mechanical constraint (FIG. 2). Uniaxial compression, especially of samples with low height-to-diameter ratio, can reduce samples to thin flakes without the onset of fracture ${ }^{32}$. Most quantitative studies of cold work have been based on rolling (FIG. 2a), where high strains are achieved by repeated passes (between the rolls), sometimes with stacked samples ${ }^{33}$; the maximum reported thickness reduction is $92 \%$ (corresponding to a normal strain of 11.50$)^{34}$. In wire-drawing (FIG. 2b) a $93 \%$ reduction of the area of the wire cross-section has been achieved ${ }^{35}$. The highest 
plastic strains have been applied by high-pressure torsion (HPT, FIG. 2c): the overall equivalent normal strain was 925 at the circumference of a sample subjected to 50 revolutions ${ }^{36}$. Surfaces can be treated by laser-shock- and shot-peening ${ }^{37,38}$ (FIG. 2d), and ribbons can be ball-milled (FIG. 2e) to achieve thickness reductions of up to $97 \%$. Plastic flow induced by these severe treatments has the potential to induce substantial changes in the structure and properties of metallic glasses.

Wires of metallic glass produced by cold-drawing can show a tensile plastic strain of up to $0.5 \%$ [REF. 35], which is noteworthy considering that metallic glasses usually lack any ductility. The presence of shear bands in the drawn wires results in relatively uniform deformation, because they facilitate the initiation of flow (the yield stress of drawn wires is reduced by as much as 35\%) and block shear-band propagation $^{35}$. Similar effects are observed after cold-rolling, which reduces both the serrations in stress-strain curves and the striated pile-ups around indentations in metallic glasses ${ }^{40}$. In rolled samples the orientation of the bands influences their plasticity $^{34,41,42}$. To improve material properties by rolling it is not necessary to induce extensive deformation: a thickness reduction of 3-5\% can result in increases in compressive plasticity, in ductility of up to $0.8 \%$ [REFS 43,44 ], and in toughness increases ${ }^{45}$.

Metallic glasses subjected to cold work are more heterogeneous ${ }^{43,46}$ and inevitably develop internal stresses. These stresses can be exploited to improve the plasticity of metallic-glass samples ${ }^{47,48}$ and have remarkable effects on properties such as hardness ${ }^{42}$.

Increased disorder, or rejuvenation, in metallic glasses is associated with a decreased coordination number of the atoms ${ }^{49}$ and an increased volume (BOX 1). This volume increase is often described in terms of 'free volume', more specifically the relative amount by which the volume exceeds that of an ideal glass that would be obtained after infinitely slow cooling. The free volume is often the order parameter in models of deformation ${ }^{22}$, and hence, the characterization of changes in volume and density can be informative. The relative density decrease resulting from cold work of as-cast glasses is comparable to the relative increase obtained upon annealing ${ }^{50}$. A Zr-based bulk metallic glass cold-rolled to achieve a thickness reduction of $63 \%$ showed a density decrease of $0.06 \%$ [REF. 50]. Larger decreases, of up to $0.3 \%$, have been reported in another case with a thickness reduction of only 3\% [REF. 43]. On initial deformation, the density can appear to increase; this is attributed to the closing of cast-in voids. If deformation is continued, the density decreases and the rate of decrease may accelerate, possibly because of the generation of nano- or micro-voids and cracks. The generation of such defects is suggested by the incomplete recovery of the initial density upon annealing ${ }^{51}$. Diffraction methods are potentially attractive 
for the estimation of free volume because the measured densities are not affected by voiding or cracking. Although a direct link between diffraction data and density changes remains elusive ${ }^{52}$, preliminary studies gave interesting results: data from a heavily deformed Pd-based bulk metallic glass plate suggested a density decrease of $0.16 \%$, whereas a Zr-based bulk metallic glass, after a similar treatment, showed a density decrease of $0.9 \%$ [REF 39], which is one of the largest decreases reported to date. Although density is a fundamental property of materials, the precision with which it can be measured is too low to enable a useful quantification of the effects of cold work; for this purpose it is better to rely on stored energy.

\section{Figure 3 near here}

Stored energy of cold work. The extent of deformation effects can be quantified in terms of increased enthalpy (FIG. 3). For polycrystalline metals in the early stages of room-temperature deformation, less than $10 \%$ of the mechanical work is stored in the metal $^{53}$. In contrast, in the initial phase of cold deformation of glassy polymer poly(methyl methacrylate), the stored energy is $30-60 \%$ of the work done ${ }^{54}$. Coldrolling a Pd-based metallic glass results in the storing of roughly $4 \%$ of the energy ${ }^{55}$, similar to the value for polycrystalline metals. For polycrystals, the maximum stored energies $^{53}$ in conventional metalworking processes are a few hundreds of $\mathrm{J}$ g-atom $^{-1}$ (1-2\% of the latent heat of melting, $\Delta H_{\mathrm{m}}$ ) for pure metals, rising to nearly $1 \mathrm{~kJ} \mathrm{~g}$ atom $^{-1}$ (5-10\% of $\Delta H_{\mathrm{m}}$ ) for solid solutions. The highest stored energy reported for polycrystals $^{53}$ is $4.0 \mathrm{~kJ} \mathrm{~g}$-atom $^{-1}$ for ordered $\mathrm{Cu}_{3} \mathrm{Au}$ wire-drawn at $77 \mathrm{~K}$. Roomtemperature ball-milling used to generate nanocrystalline metals gave a stored energy as high as $7.7 \mathrm{~kJ} \mathrm{~g}$-atom $^{-1}$ for Ru [REF. 56]. In these last two cases, the energy was roughly $30 \%$ of $\Delta H_{\mathrm{m}}$, which may represent a practical maximum for energy storage in the crystalline state (FIG. 3). If intermetallic compounds are amorphized under deformation $^{57,58}$, even higher energies can be stored as a result of the phase change.

\section{Figure 4 near here}

The increased enthalpy of deformed polycrystalline metals arises from the defects induced in the crystalline structure. It could be thought that achieving a similar effect in metallic glasses would be difficult because of their non-crystalline, and apparently disordered condition. Metallic glasses are ordered, however, and the entropies of the glasses can be almost as low as those of their crystalline counterparts ${ }^{4,59}$. Metallic glasses do show excess enthalpy - the heat of crystallization of a relaxed glass, or of a supercooled liquid near $T_{\mathrm{g}}$, is about $40 \%$ of $\Delta H_{\mathrm{m}}$ [REF. 56]. In comparison to the 
relaxed state, as-cast metallic glasses have a significant excess enthalpy, characterized as the heat of relaxation, $\Delta H_{\text {rel }}$, measured by heating the sample to $T_{\mathrm{g}}$ in a calorimeter (FIG. 4a). The range of $\Delta H_{\text {rel }}$ for rapidly quenched (melt-spun) metallic glasses goes from $600 \mathrm{~J} \mathrm{~g}$-atom $^{-1}$ to $5.5 \mathrm{~kJ}$ g-atom ${ }^{-1}$ [REF. 60]; we take $1-1.5 \mathrm{~kJ} \mathrm{~g}$-atom $^{-1}(11-17 \%$ of $\left.\Delta H_{\mathrm{m}}\right)$ as a representative value. For bulk metallic glasses (cooled more slowly), a representative value is $300 \mathrm{~J} \mathrm{~g}$-atom $^{-1}$ (3\% of $\Delta H_{\mathrm{m}}$ ) [REFS 36,42] (FIG. 3). For these estimations, we use the information that $\Delta H_{\mathrm{m}}$ is in the range from 5 to $12 \mathrm{~kJ}$ g-atom ${ }^{-1}$, and we take $9 \mathrm{~kJ} \mathrm{~g}$-atom $^{-1}$ as a representative value if a range of metallic glasses is being considered, or if no other value is available. Unlike in the case of polycrystalline metals, the range of initial enthalpies needs to be taken into account to quantify the effects of cold work on metallic glasses.

Heavy deformation, for example by cold-rolling to achieve a 50-60\% thickness reduction, results in an increase of $\Delta H_{\text {rel }}$ by $300-450 \mathrm{~J}$ g-atom ${ }^{-1}$ (3-5\% of $\Delta H_{\mathrm{m}}$ ) [REFS 42,51,55,61] (FIG. 4a). The highest reported increase (apparently not yet at saturation) is $1,770 \mathrm{~J} \mathrm{~g}$-atom $^{-1}$ for a $\mathrm{Zr}$-based bulk metallic glass, after application of a normal strain of 925 . The sample, subjected to high-pressure torsion, had a total $\Delta H_{\text {rel }}$ of 2,060 J g-atom ${ }^{-1}$ (24\% of $\Delta H_{\mathrm{m}}$ ) [REF. 36]. A similar value may be reached in coldrolled melt-spun ribbons ${ }^{61}$. Thus, the maximum energy (normalized as a fraction of $\left.\Delta H_{\mathrm{m}}\right)$ stored in cold-worked metallic glasses is similar to that stored in polycrystalline metals. However, in addition to this energy, there is the excess enthalpy represented by the heat of crystallization. As a result, metallic glasses that underwent extreme cold work may have energies that are higher than the energy of the annealed crystalline state by approximately $65 \%$ of $\Delta H_{\mathrm{m}}$ (FIG. 3 ).

The structural effects of cold work have to be considered in terms of inhomogeneous flow. The heavily deformed material inside the shear bands displays effects such as a greatly increased atomic diffusivity ${ }^{62}$, but the volume fraction occupied by the bands is low, even in heavily deformed bulk samples. For example, 1-mm-thick plates of Zr-based bulk metallic glasses show shear-band spacings that decrease on continued rolling, but reach a minimum at 30-40 $\mu \mathrm{m}$ [REF. 42]. If the thickness of a shear-band is around 10-20 nm, the volume fraction occupied by shearbands is $0.03-0.07 \%$. In thinner samples, for example in a $22 \mu \mathrm{m}$ ribbon, rolling gives shorter spacings, of around $100 \mathrm{~nm}$, and hence the volume fraction of material occupied by shear bands is higher, but still under $20 \%$ [REF. 63].

From early studies it was clear that if both enthalpy and volume increases in coldworked metallic glasses were assumed to arise only from changes within the shear bands, these changes would have to be unreasonably large. This can be better quantified looking at a single shear band ${ }^{64}$. An 80- $\mu$ m-thick slab of a Zr-based bulk metallic glass, cut to include the shear band in the central plane, shows a cold-work 
enthalpy of $1.7 \mathrm{~kJ}$ g-atom ${ }^{-1}$. However, if the cold-work enthalpy were attributed solely to a 20-nm-thick band, the stored energy in the band would be roughly 6.8 MJ g-atom ${ }^{-1}$, nearly $10^{3}$ times a typical heat of melting. A similarly unphysical conclusion is reached when considering volume changes. It is undeniable that the volume fraction of the material affected by the deformation must exceed that thought to be occupied by shear bands. The profiles of reduced hardness across a shear band suggest a shear-affected zone with a thickness that is of the same order of magnitude as the length of shear offset on the band ${ }^{64,65}$. This observation makes it easier to interpret the enthalpy and volume increases that result from deformation, but it remains unclear how this effective thickness relates to the flow pattern in shear-band operation.

Our focus here is on stored energy, but most of the work done on deformed metallic glasses is not stored but dissipated as heat, in a process that is likely to be near-adiabatic. As a consequence of the high flow stresses of metallic glasses, the work done must provide substantial heating. There have been few studies of such effects. The heating that arises in samples during shot-peening appears to promote stress relaxation at longer peening times ${ }^{47}$, and it is likely to contribute to the decrease in $\Delta H_{\text {rel }}$ when an as-cast glass is peened ${ }^{38,66}$. In cold-working, increases of tens of degrees in the overall sample temperature can be expected, through accelerated relaxation, to reduce the extent of rejuvenation. In shear bands much higher temperature increases may occur over very short times. Outside the bands, the effects of these temperature increases are realized only very close by ${ }^{23}$. The main effect would thus be observed within the bands, and the residual temperature rise after shear might enable partial dissipation of the excess energy stored during cold work.

It is intriguing to investigate the parameters that set the upper limit of the stored energy. In a highly deformed metallic glass, the balance between damage and relaxation would lead to a steady-state degree of rejuvenation (BOX 2), but other effects may prevent reaching this steady state. Similarly to the manner in which recrystallization may set a limit of how much cold-work energy can be stored in a polycrystalline metal, structural transformations may limit the energy-storage ability of deformed metallic glasses. The excess volume in the glass may start to condense forming voids, or the glass may crystallize. Both phenomena have been observed in shear bands ${ }^{23,63}$, and crystallization of various kinds has been observed also outside shear bands ${ }^{67,68}$. The stability of the deformed state can be evaluated in terms of other properties. For cold-rolled metallic glasses, the Young modulus is reduced by $1-8 \%$ [REFS 34,44], but for the extreme deformation obtained by high-pressure torsion discussed above ${ }^{36}$ the Young modulus (measured by nanoindentation) is reduced by a remarkable $30 \%$. 
Hot-working and viscous flow. An important feature of metallic glasses is their mouldability when heated into the supercooled liquid state, above $T_{\mathrm{g}}$. The homogeneous flow of the viscous liquid, exploited for example in blow-moulding ${ }^{69}$, makes metallic glasses attractive for the manufacture of small components. Fine shapes or surface patterns, even down to the nanometre scale, can be produced ${ }^{70}$; the formation of these is facilitated by the lack of microstructure in the glass. To avoid crystallization, the processing time above $T_{\mathrm{g}}$ must be short, and to this end, techniques such as pulse-current heating have been developed ${ }^{71}$. Processing in such short times may require rapid flow of the supercooled liquid, which may reduce its viscosity - an effect called 'shear thinning, ${ }^{72}$. The increased mobility in the resulting nonNewtonian flow (FIG. 1b) reduces the time required for the onset of crystallization compared to that of the liquid without shear at the same temperature. The reduction is stronger at higher shear rates and lower temperatures ${ }^{73,74}$. It is not clear if the excess volume associated with this high-temperature homogeneous flow can be frozen in to give a rejuvenated glass in the same way as for the material in shear bands. However, rejuvenation can be achieved by flow (creep) just below $T_{\mathrm{g}}$ [REF. 75]. Structural changes detected by high-energy X-ray diffraction suggest that rejuvenation is largely associated with anelastic strain $^{76}$, as discussed below in the context of induced anisotropy.

\section{Box 2 | Energetic processing of metals}

The mechanical deformation of solids transfers energy to the material. This energy transfer also occurs in other processing methods including irradiation. Energetic processing of this type can lead to an energy increase in the sample (rejuvenation), but also to relaxation. These opposing trends are important for metallic glasses, which show a range of energies in their as-cast state (BOX 1). After long processing times, the changes induced in the solid's structure and properties eventually saturate. More specifically, a steady state is reached in which the rate of structural change (damage) introduced by the processing is balanced by the rate of structural relaxation, which is enhanced by the increased atomic mobility in the processed material. In the steady state, enthalpy, entropy, and volume are higher - that is, the state is less relaxed, more rejuvenated, at higher processing rates (strain rate or irradiation flux) and at lower temperatures. This reflects the balance of damage and relaxation rates.

\section{Box 2 Figure near here}


The illustration shows three possible cases: (a) a solid of low initial energy (for example, a polycrystal with a low dislocation density or a relaxed glass), retains some of the injected energy, and evolves into a more rejuvenated state. (b) a solid of high initial energy (for example, a polycrystal with a high dislocation density or a rapidly quenched glass) evolves into a more relaxed state. (c) a given initial state may evolve in either direction, depending on processing rate and temperature. Such considerations, which are well understood for mechanical deformation ${ }^{53}$ and irradiation $^{146}$ of polycrystalline metals, need to be further explored in the context of the mechanical deformation of metallic glasses.

\section{Effects of elastic and thermal strains}

Relaxation. Plastic deformation of metallic glasses can induce substantial changes in their structure and properties. This is not unexpected considering the effects of deformation on polycrystalline metals. It is surprising, however, that in metallic glasses small strains (considerably smaller than at the onset of general yielding) can induce changes that are of similar magnitude to those induced by large plastic strains. This behaviour can be interpreted by considering the nature of elastic and anelastic deformations in metallic glasses. The fact that small elastic deformations can have substantial effects was realized when it was shown that ultrasonic vibrations applied to a metallic glass near its $T_{\mathrm{g}}$ accelerated crystallization ${ }^{77}$. If applied at room temperature, ultrasonic vibrations accelerate structural relaxation rather than crystallization $^{78}$, and the effects of this treatment include a $14 \%$ increase in hardness.

Mechanical tests involving nanoindentation show that elastic loading affects metallic glasses. Upon loading, the onset of shear banding is indicated by a sharp 'pop-in' that is characterized by an increase in the indentation depth, $h$, on a load versus $h$ curve. The load at which the first pop-in occurs shows a distribution of values, which indicates heterogeneity in the glass ${ }^{79}$. When the indenter is used to impose loading cycles, over a threshold amplitude and number, and at loads significantly below that for the first pop-in (that is, in the elastic range), indentation shows a narrower distribution of pop-in loads (indicating reduced heterogeneity in the sample) and a hardening of the material quantified as load values increased by $\sim 20 \%$ (FIG. 4b). This increase in yield load has been interpreted as an evolution toward an 'ideal glass' configuration of higher structural order ${ }^{80}$. If there is a pause between pre-cycling and indentation, however, the hardening is substantially reduced ${ }^{81}$, suggesting that the hardening effect is at least partly due to anelastic strain accumulated under the indenter tip, rather than to a relaxed denser glassy structure. 
Molecular dynamics (MD) studies can simulate the hardening process and show that crystallization can occur in the later stages of cycling, but structural change in the glassy phase itself has not been characterized ${ }^{82,83}$.

A complex multiaxial stress state develops under a spherical nanoindenter tip ${ }^{82}$. It is interesting to check whether similar hardening effects attributable to cyclic loading arise in simpler stress states in macroscopic bulk metallic glasses. Such effects were first detected in fatigue tests. In high-cycle fatigue testing of polycrystalline metals, application of a gradually increasing amplitude of stress can result in increased fatigue strength, an effect known as 'coaxing'. Although the underlying mechanisms must be different to those in crystalline alloys, a Zr-based bulk metallic glass shows a strong coaxing effect, without the loss of toughness that would be expected on annealing ${ }^{84}$. In metallic glasses, as in polycrystalline alloys, the coaxing effect implies structural change. Indeed, structural changes in fatigue-tested Zr-based bulk metallic glasses have been detected using neutron scattering ${ }^{85}$. Thermal vibrations obscure the effect at room temperature, but diffraction at $10 \mathrm{~K}$ detects permanent changes in the pair distribution function, which are associated with a reduced volume and a lowered vibrational density of states $^{85}$.

Structural change on cyclic loading implies that there is energy dissipation even in the elastic regime. This is shown in cyclic compressive loading of micropillars: more specifically, as the loading and unloading rates are increased, hysteresis occurs ${ }^{86}$. Similar effects have been seen for cyclic loading of melt-spun ribbons and bulk metallic glasses ${ }^{87,88}$. They have been associated with ' $\beta$ relaxation' (the lowertemperature mode out of the two main relaxation modes in glasses ${ }^{87,89}$, FIG. 4a), an increase in density, and even incipient crystallization ${ }^{88}$ at room temperature.

The results so far indicate that elastic loading can accelerate relaxation. Similar acceleration of relaxation may be possible by plastic deformation. Although this effect is usually overwhelmed by the generation of free volume on shear, it is observable when shear banding is avoided - this avoidance has been achieved in tensile tests on samples with a thickness of $400 \mathrm{~nm}$ and widths as low as $210 \mathrm{~nm}$. In samples with such small cross-sections, nucleation of shear bands is suppressed, and the elastic strain limit (up to $4.4 \%$ ) is higher than for macroscopic samples ${ }^{90}$. Microscopic samples can be tested beyond the elastic limit, and upon unloading and re-loading they show apparent work-hardening. This is interpreted in terms of the progressive activation of shear transformation zones with increasing activation energy: initial loading activates the lowest-energy shear transformation zones, which cannot shear again on subsequent reloading ${ }^{90}$. A similar effect is observed upon tensile loading of a deeply notched specimen when the triaxial stress state favours diffusion over shear ${ }^{91}$. The gauge length of the metallic glass shows stable flow and hardening, which is 
consistent with densification. It was suggested that similar relaxation would not arise in compressive flow, because atomic rearrangements would be retarded by the hydrostatic compressive stress component ${ }^{91}$. The hydrostatic component of stress can indeed have thermodynamic as well as kinetic effects. Unsurprisingly, annealing under pressure favours glassy states of higher density and hardness ${ }^{92}$.

We have seen that very different applications of elastic strains can result in hardening. Whereas some of these effects can be attributed to a relaxation to lowerenergy states, others seem likely to be a consequence of higher-energy states associated with accumulated anelastic strain. A better differentiation of these effects remains a key issue that needs to be addressed.

Rejuvenation. Rejuvenation in the nominally elastic regime was first demonstrated in uniaxial compressive loading of a sample of a $\mathrm{Ni}_{62} \mathrm{Nb}_{38}$ metallic glass at $95 \%$ of the macroscopic yield stress ${ }^{93}, \sigma_{\mathrm{y}}$. After 30 hours of loading at room temperature, which induced a creep strain of $-0.36 \%$, the compressive plasticity was dramatically improved, from essentially $0 \%$ in as-cast samples to $-5.2 \%$. Experimental and computational studies on a variety of metallic glasses loaded at stresses as low as 0.8 $\sigma_{\mathrm{y}}$ demonstrated that such 'elastostatic' compression treatments result in reduced density and elastic moduli, and increased enthalpy ${ }^{94,95}$. These are all signs of disordering and rejuvenation, the degree of which can be quantified by the heat of relaxation $\Delta H_{\text {rel }}$. The maximum increase induced in $\Delta H_{\text {rel }}$ so far is the saturation value of $251 \mathrm{~J} \mathrm{~g}$-atom ${ }^{-1}$ for $\mathrm{Cu}_{57} \mathrm{Zr}_{43}$ loaded at $0.85 \sigma_{\mathrm{y}}$ at $77 \mathrm{~K}$ (the saturation value is obtained after a long loading time $)^{96}$. In this case, the relaxation spectrum is clearly bimodal (FIG. 4a) and possibly associated with $\beta$ relaxation ${ }^{89,96,97}$. This saturation value is higher than that obtained for loading at room temperature, an expected consequence of impeded relaxation at lower temperature (BOX 2). The most striking observation is that this value is of similar magnitude to those induced by plastic deformation. To attain the same enthalpy increase $\left(251 \mathrm{~J}_{\mathrm{g} \text {-atom }}{ }^{-1}\right)$ by cold-rolling ${ }^{55}$ would require a reduction in thickness of $\sim 50 \%$. It might be tempting to treat these increases in $\Delta H_{\text {rel }}$ as stored energy, in the same way as for plastic deformation, but these enthalpy increases greatly exceed the mechanical work done-the creep strains are small. We can conclude that the creep-induced disordering of the metallic glass, obtained on loading at room temperature and below, is an endothermic process that draws heat from the surroundings ${ }^{97}$. Rejuvenation is also expected to increase atomic mobility; thus such effects may underlie the partial crystallization sometimes observed in elastostatic loading ${ }^{98}$.

Equal-channel angular pressing (FIG. 2f) can be adapted, using an internal die angle $\phi=172^{\circ}$, to apply stresses just below $\sigma_{\mathrm{y}}$ to a bulk metallic glass plate. Repeated 
elastic loading (6 passes through the die) rejuvenated a Zr-based bulk metallic glass so that the $1.2 \%$ compressive plasticity of the as-cast state was improved to $10 \%$ [REF. 99].

It is reasonable to wonder whether thermal strains can have effects similar to those of elastic strains. A preliminary study ${ }^{100}$ showed that cycling between room temperature and $77 \mathrm{~K}$ induces rejuvenation; a melt-spun $\mathrm{La}_{55} \mathrm{Ni}_{20} \mathrm{Al}_{25}$ glass showed a maximum $\Delta H_{\text {rel }}$ increase of $340 \mathrm{~J} \mathrm{~g}$-atom $^{-1}$ after 10 cycles (FIG. 4a). Correspondingly, the cumulative distribution curve for initial yield pressure under a nanoindenter tip was shifted to values some $20 \%$ lower (FIG. 4b). Bulk metallic glasses of different compositions showed, after cycling, reduced microhardness, increased shear-band population density and increased plasticity under uniaxial compression. The increases in $\Delta H_{\text {rel }}$ are even higher than for elastostatic loading (FIG. 3); similarly they must be attributed, as discussed above, to induced endothermic disordering ${ }^{97}$. Thermal cycling enabled the restoration of plasticity in a sample embrittled by annealing ${ }^{100}$; rejuvenation by thermal cycling is particularly attractive because it can be applied to any sample geometry and is intrinsically isotropic.

The linear thermal strain generated by cooling from room temperature to $77 \mathrm{~K}$ is around $-0.2 \%$. Mild processing, such as cycling over that temperature range or elastostatic loading to creep strains less than $-0.4 \%$, can induce large increases in $\Delta H_{\text {rel }}$, suggesting that non-affine strains in the nominally elastic regime are rather efficient in inducing disordering through local rearrangements. Further studies are needed to gain a more detailed understanding of the effects of non-affine strains.

Cyclic loading. Mechanical cycling has been comprehensively studied in MD simulations of model atomic glasses. Cycling the strain ultimately leads to a steady state and, depending on the initial state, the glass is relaxed or rejuvenated ${ }^{101,102}$ (BOX 2). For low-amplitude strain, relaxation is the most likely outcome, and the glass returns to the same energy minimum at the end of each cycle. In this regime, the cyclic loading can be regarded as 'training', possibly recording multiple 'memories' in the glass ${ }^{103}$. Above a threshold amplitude that is close to the yield strain, rejuvenation is more likely. There is a sharp increase in the energy dissipation, and the memory of the initial state of the glass is lost ${ }^{102,103}$. In real metallic glasses, training (ageing) may happen in the processes discussed above: hardening because of cyclic nanoindentation loading ${ }^{80}$, and fatigue coaxing ${ }^{84}$. Cyclic loading in the plastic regime, which leads to strong rejuvenation, is not easily realized for metallic glasses. Nevertheless, if optimized (in terms of temperature, frequency and amplitude), cyclic loading holds a great and largely unexplored potential for accessing new relaxed and rejuvenated states. Metallic glasses may respond to cyclic loading in a similar way to 
dense colloids, foams and granular materials ${ }^{102,103}$. Granular materials can show compaction (analogous to ageing) or rejuvenation, depending on the intensity of mechanical agitation ${ }^{101}$. The fact that dense atomic systems such as metallic glasses may show an analogous behaviour is a fascinating possibility.

\section{Induced anisotropy}

The glassy state cannot be fully specified by properties such as enthalpy because these properties fail to describe, for example, the degree of anisotropy. A liquid, unless composed of molecules capable of being oriented, is expected to be isotropic, but a glass formed from the liquid can be anisotropic. Metallic glasses produced by meltspinning $^{8}$, which involves strong shear, show anisotropy in several aspects ${ }^{104-106}$. Anisotropy can also be induced in metallic glasses after production. For example, annealing in a magnetic field induces uniaxial anisotropy ${ }^{107}$, and, if stress is concurrently applied, the development of anisotropy is greatly accelerated ${ }^{108}$, presumably because the generation of free volume increases mobility. In addition, induced anisotropy has been shown in tensile strength ${ }^{109}$, elastic constants ${ }^{110,111}$ and plasticity $^{75}$.

\section{Figure 5 near here}

In most cases, induced anisotropy is reversible and can be attributed to stored anelastic strain, as was quantified in a structural study ${ }^{112}$. Using high-energy X-rays from a synchrotron source, diffraction patterns can be obtained in transmission. An isotropic sample gives a circular first halo in the structure function, $S(Q)$, which becomes elliptical if anisotropy is induced by elastic strain under load or by anelastic strain frozen-in after creep loading ( $Q$ is the magnitude of the scattering vector). The ellipticity is best detected in the difference between the patterns obtained from the sample in two orthogonal orientations ${ }^{113}$ (FIG. 5a). The degree of anisotropy can be quantified as the relative difference $\Delta Q / Q$ between the major and minor axes of the first-halo ellipse in $S(Q)$. In the example in FIG. $5 \mathrm{a}, \Delta Q / Q \approx 7 \%$, which is a typical magnitude for samples that have been deformed homogeneously with creep strains of a few percent. Samples deformed to much greater extents, with creep elongations of over $70 \%$, show much lower diffraction anisotropies $(\Delta Q / Q \approx 0.3 \%)$ that decrease with increasing strain [REF. 114]. Inhomogeneously deformed metallic glasses also show anisotropy in their diffraction patterns, but the effects behind their anisotropy are more complex ${ }^{113}$.

The stress-induced magnetic anisotropy in Co-based metallic glasses changes sign as a function of annealing temperature ${ }^{115,116}$. A similar reversal is observed in 
the elastic anisotropy induced in a Pd-based bulk metallic glass by compressive uniaxial creep ${ }^{110}$. At small strains (up to about $-10 \%$ ) the anisotropy has one sign and is reversible - annealing without stress erases it — but at larger strains it changes sign (FIG. 5b) and becomes irreversible. These two regimes can be associated, even more directly for elastic than for magnetic anisotropy ${ }^{115}$, with the predominant influence of stored anelastic strain or of viscous flow ${ }^{110}$, respectively. The sign of the anisotropy associated with each regime is reversed if the pre-treatment creep is tensile rather than compressive ${ }^{111}$.

Stored anelastic strain must induce anisotropy, but this is not always true for viscous flow. In Newtonian flow no anisotropy is induced, but at higher strain rate flow-induced structural change leads to non-Newtonian flow and to anisotropy ${ }^{111}$.

In polymers, tensile creep induces alignment of the chains, and therefore of covalent bonds, in the direction parallel to the tensile axis. The nature of the structural anisotropy in metallic glasses, first explored in X-ray diffraction experiments ${ }^{117}$, appears to be different because, with metallic bonding, nearestneighbour identity is more easily interchanged. Under tensile stress, local atomic configurations show severing of bonds in the axial direction and formation of new bonds in the transverse plane. These rearrangements relax the system under stress, but induce anisotropy when the stress is released. The net effect is a creep-induced 'bond orientational anisotropy' that is detectable in the anisotropy of $S(Q)$ (FIG. 5a). The structural basis for anisotropy may include not only topological but also chemical ordering: for example, in ferromagnetic metallic glasses, there may be directional ordering of $\mathrm{Fe}-\mathrm{Fe}$ pairs ${ }^{105}$.

For Zr-based bulk metallic glasses subjected to creep, rejuvenation roughly balances the structural relaxation induced by annealing. Samples that are brittle ascast or after annealing show improved plasticity after creep, but these effects are clearly anisotropic and go beyond what can be explained only in terms of increased free volume ${ }^{75}$. Inducing axial plasticity by high-temperature compressive creep has effects similar to those of elastostatic loading at room temperature; in the latter case anisotropic effects can be expected, but have not yet been explored.

Metallic glasses subjected to small creep strains develop an anisotropy that can be interpreted in terms of frozen-in anelastic strain. After larger creep strains, the anisotropy in the properties of metallic glasses can change sign and increase, yet the anisotropy observed in diffraction experiments is weak. The nature of anisotropy induced by viscous flow requires further study, and may be relevant for the understanding of deformation mechanisms. 


\section{Alternatives to mechanical processing}

Irradiation. The effects of irradiation of various kinds-electrons, protons, thermal and fast neutrons, light and heavy ions - on metallic glasses have been widely studied in experiments and simulations. The damage and mobility that irradiation induces have many parallels with the effects of mechanical deformation. Metallic glasses appear to be immune from the unlimited void swelling observed in some crystalline alloys ${ }^{118}$, which suggests their suitability for nuclear applications ${ }^{119,120}$. Irradiation can induce relaxation or rejuvenation, depending on the initial state of the glass and on the irradiation conditions (BOX 2). Under given conditions, the same steady state can be approached from both lower- and higher-energy states ${ }^{119}$, and the steady-state energy is higher under more intense irradiation ${ }^{121}$. Irradiation can lower the energy of glasses not only by structural relaxation ${ }^{122}$, but also by partial nanocrystallization ${ }^{123-}$ 125

Rejuvenation of metallic glasses by irradiation reduces the yield stress and the softening after yield, and promotes a transition from shear banding to a more homogeneous flow ${ }^{118,119,121,126-128}$. Irradiation can restore plasticity in glasses embrittled by annealing, and the extent of damage required $\left(10^{-5}\right.$ to $10^{-2}$ displacements per atom) depends both on the degree of relaxation of the glass prior to irradiation and on the nature of the radiation ${ }^{129}$. The improvement in plasticity is associated with a reduction in density ${ }^{118,119,126,128,129}$, which saturates at $\sim 1 \%$. The effects of irradiation are seen also in structural studies, which typically show disordering $^{121,128}$, and in changes in the superconducting ${ }^{126}$ and magnetic properties ${ }^{120,125}$ of the samples.

High-energy primary knock-on atoms induce a cascade of collisions, giving a thermal spike in which regions of the sample roughly $10 \mathrm{~nm}$ wide reach temperatures much higher than $T_{\mathrm{m}}$ for a few picoseconds. MD simulations show that the glassy states reached after irradiation are very similar to those reached by ultra-rapid quenching at $10^{12}$ to $10^{14} \mathrm{~K} \mathrm{~s}^{-1}$ [REFS 118,119,121]. For example, $0.5 \mathrm{MeV} \mathrm{Nb}$ primary knock-on atoms, after a dose of > 0.003 displacements per atom, take simulated $\mathrm{Cu}-\mathrm{Nb}$ glasses to a steady state nearly indistinguishable from that reached by quenching the liquid at $6 \times 10^{13} \mathrm{~K} \mathrm{~s}^{-1}$ [REF. 118]. The damage mechanisms may be different at lower particle energies ${ }^{119,124,129}$, but similar final states are reached.

Physical vapour deposition. Physical vapour deposition (PVD) is a powerful technique that gives access to different glassy structures. The technique has mostly been applied to organic materials, for which the main interest lies in accessing 'ultrastable' glasses, that is glasses with exceptionally low energies ${ }^{130,131}$. As a 
reference we can consider a glass formed at a particular $T_{\mathrm{g}}$ by cooling a liquid at a given rate. If physical vapour deposition is used to form a thin film of the same substance on a substrate at a temperature $T_{\text {sub }}=T_{\mathrm{g}}$, then the thin film has essentially the same structure as the glass formed from the liquid. If the deposition is onto a colder substrate, however, a denser structure can be formed, because the molecular mobility on the surface during deposition is much higher than in the bulk. The densest glass-like deposits are obtained for $T_{\text {sub }}=(0.85-0.80) T_{\mathrm{g}}$ : their properties are consistent (apart from some anisotropy-related features) with ageing for $10^{3}$ to $10^{9}$ years; thus they are not practically accessible by liquid cooling ${ }^{130}$. Metallic glasses deposited by magnetron sputtering at low rates are ultrastable in deposits up to $30 \mu \mathrm{m}$ thick $^{132-134}$. In contrast, for pure-metal systems, at $T_{\text {sub }} \ll T_{\mathrm{g}}$ the reduced surface mobility leads to unstable deposits that can be regarded as highly rejuvenated; the degree of rejuvenation sustainable at a given temperature is limited by crystallization $^{11}$.

Condensation. A method related to PVD is the inert-gas condensation of nanometrescale spheres of metallic glass, followed by the collection of the spheres and their consolidation under pressure to form a 'nanoglass'. MD simulations show that the original spheres remain identifiable as a consequence of their high density relative to the interfaces between them, and upon annealing the interfaces become blurred but the overall free volume remains high ${ }^{135}$. Experimental studies of a $\mathrm{Sc}_{75} \mathrm{Fe}_{25}$ nanoglass reveal 10-nm-diameter glassy 'grains' separated by 1-nm-thick softer interfaces ${ }^{136,137}$. In compression and tensile tests on micro-pillars, the nanoglasses show substantially better plasticity than melt-spun ribbons; in particular, samples with a $400 \mathrm{~nm}$ crosssection exhibit a tensile plasticity of up to $15 \%$, consistent with the more homogeneous flow suggested in simulations ${ }^{136,137}$. This promotion of homogeneous flow $^{138}$ is related not only to the overall high free volume, but also to the inhomogeneous structure of the nanoglass, which results in the generation and intersection of multiple shear bands in the soft interfaces. The term nanoglass has been applied also to materials made by methods different from inert-gas condensation and consolidation. High-pressure torsion (FIG. 2c) gives rejuvenated bulk metallic glasses, and permits to obtain nanoglass-like structures in bulk samples, enabling, for example, calorimetric measurements ${ }^{139}$. Although nanoglasses are principally of interest for their high degree of rejuvenation, similar materials made by sputterdeposition ${ }^{140}$ have also been associated with ultrastable states ${ }^{134}$. 


\section{Accessible glassy states in the bulk}

Relaxed states. As already noted, the discovery of ultrastable organic glasses demonstrates that deposition from the vapour can give access to relaxed states not realized before ${ }^{130}$. Preliminary results hint at similar effects in metallic glasses ${ }^{132-134}$, but these studies are restricted to thin coatings, and ultrastable states obtained by deposition are likely to be anisotropic.

As discussed above, ultrasound accelerates structural relaxation and crystallization $^{77,78}$. The degree of acceleration is higher at lower temperature, and crystallization times can be shortened by several orders of magnitude ${ }^{141}$. Thus, at optimized temperatures, ultrasonic and related treatments may allow access to deeply relaxed states not otherwise reachable in bulk materials. Such substantial relaxation has yet to be realized, but research on the optimization of these treatments remains in its infancy.

Rejuvenated states. Extreme thermal treatments, for example nanosecond electrical pulses applied through two nanotips which are just in contact ${ }^{142}$, can generate small liquid volumes in which the cooling rates are as high as $\sim 10^{14} \mathrm{~K} \mathrm{~s}^{-1}$. Similar states of rejuvenation but, importantly, in larger volumes, can be attained by irradiation. Ion irradiation can penetrate depths of a few micrometres ${ }^{127}$, but at low energies this can be as shallow as a few nanometres ${ }^{121,143}$. Despite these short penetration depths, useful property changes, including improvements in plasticity, can be realized in nanowires ${ }^{121,127,143}$. Similar changes can be induced in macroscopic samples using neutron irradiation, though the limited access to facilities and the activation of samples through transmutation to radioactive nuclides are both problems. Nanoglasses which have been obtained by inert-gas condensation and consolidation can be in highly rejuvenated states, but are not readily produced in bulk. Mechanical treatments of bulk glasses can be simple to apply, and are therefore of particular interest.

The maximum stored energy reported in a bulk sample after cold work is 2,060 J g-atom $^{-1}$ for a Zr-based metallic glass subjected to high-pressure torsion ${ }^{36}$. This excess energy over that for a fully relaxed glass, when considered on an enthalpy plot (see BOX 1), implies a $T_{\mathrm{g}} \sim 27 \%$ higher than that of a fully relaxed glass (that is, for a cooling rate of $0.33 \mathrm{~K} \mathrm{~s}^{-1}$ ). Given the temperature dependence of the viscosity of typical metallic-glass-forming melts ${ }^{144}$, this corresponds to a cooling rate of $10^{7}$ to $10^{8}$ $\mathrm{K} \mathrm{s}^{-1}$. In other words, the deformed bulk metallic glass is rejuvenated to an extent that exceeds that of typical melt-spun ribbons (of a few tens of $\mu$ m thick), which are known to have excellent plasticity. The clear advantage of the mechanical treatment 
is that the rejuvenated state can be obtained in samples with much larger crosssections.

We described above studies conducted on a single shear band ${ }^{64}$. From the profile of excess enthalpy across a band in a Zr-based bulk metallic glass it was estimated that in the centre the maximum excess enthalpy was $4.14 \mathrm{~kJ}$ g-atom ${ }^{-1}$. This represents $\sim 35 \%$ of $\Delta H_{\mathrm{m}}$, implying a $T_{\mathrm{g}} \approx 39 \%$ higher than that of the relaxed glass, and a structure equivalent to that which would be obtained by quenching at $\sim 10^{9} \mathrm{~K} \mathrm{~s}^{-1}$. Perhaps, with more intense plastic deformation, such a structure could be obtained in the whole bulk sample, not just near the central plane of the shear bands. As these excess enthalpies are obtained for samples deformed at room temperature, rather than at cryogenic temperatures, it is likely that these values are still far from the limit of what is possible. In studies to date, elastostatic loading ${ }^{96}$ and thermal cycling ${ }^{100}$ have given lower excess enthalpies, but for these treatments the processing conditions for rejuvenation have not yet been optimized ${ }^{100}$.

Work-hardening. Shear banding and the lack of ductility limit the exploitation of the otherwise attractive mechanical properties of metallic glasses. Shear banding can be suppressed in samples of small volume ${ }^{90,91,145}$, and this suppression would be desirable in bulk samples also. The origin of shear banding lies in the instability of work-softening discussed in connection with FIGs. 1b,c. If rejuvenation changed the glass behaviour so that it followed curve (ii) in FIG. 1c, there would be no shear banding, but tensile samples would fail by immediate necking in the plastic regime, which is associated with dilatation under shear. However, if the initial volume of a metallic glass is so high ( $\mathrm{BOX} 2$, case b) that the volume decreases under shear, then work-hardening could be expected, and it would impart ductility (resistance to necking). Metallic glasses rejuvenated by various routes show improved plasticity, with higher population densities of shear bands and failure that is more progressive, less catastrophic. MD simulations ${ }^{119,121,128}$ and experiments ${ }^{127}$ suggest that the extreme rejuvenation induced by irradiation can cause a transition from shear banding to homogeneous flow, at the cost of a significant softening of the glass. A key question, as yet unanswered, is whether a bulk glass so highly rejuvenated as to show work-hardening would be stable, in particular against crystallization.

\section{Conclusions and prospects}

Using different production and processing routes, a wide range of states is attainable in metallic glasses. The glassy states that are most rejuvenated-those with the highest energy — have been achieved by quenching nanoscale volumes of liquid and by irradiation. The states that are most relaxed — with the lowest energy-have been 
attained by physical vapour deposition of thin films. Thermomechanical processing of metallic glasses has not yet granted access to the full range of possible states. Of all available processing routes, however, thermomechanical processing offers the best prospect of achieving extreme states in bulk samples that would permit exploitation of the improved material properties.

Plastic deformation of samples at room temperature has led to rejuvenated glassy states energetically equivalent to those quenched from the liquid at nearly $10^{8} \mathrm{~K} \mathrm{~s}^{-1}$, and states equivalent to those quenched at $10^{9} \mathrm{~K} \mathrm{~s}^{-1}$ may be attainable. Significant rejuvenation can also be achieved (well within the nominally elastic range) by applying stresses or temperature changes; these surprising results suggest that nonaffine strains are efficient in inducing damage in metallic glasses, a finding that has not yet been exploited. Annealing-induced embrittlement - a key problem of metallic glasses - can be reversed by rejuvenation. This is an example of the type of benefit that thermomechanical treatments might bring. However, these treatments, especially when they include features such as cyclic loading, await optimization: the range of glassy states that can be achieved has so far been explored only to a limited extent.

Highly rejuvenated states are interesting to pursue as they may offer a route to work-hardening of metallic glasses that could transform the applicability of these materials. It is not yet clear whether such states can be achieved in bulk, structurally stable glasses, but thermomechanical processing offers good prospects of achieving this goal.

In addition, thermomechanical processing gives access to anisotropic states of fundamental and practical interest. Anisotropy in metallic glasses is important in influencing their magnetic properties, but its role in optimizing their mechanical properties remains unexplored; it is possible that studies of anisotropy will play a key role in understanding the mechanisms of plastic deformation.

\section{REFERENCES}

1. Klement, W., Willens R. H. \& Duwez, P. Non-crystalline structure in solidified gold-silicon alloys. Nature 187, 869-870 (1960).

2. Takeuchi, A. \& Inoue, A. Classification of bulk metallic glasses by atomic size difference, heat of mixing and period of constituent elements and its application to characterization of the main alloying element. Mater. Trans. 46, 2817-2829 (2005).

3. Inoue, A. \& Takeuchi, A. Recent development and application products of bulk glassy alloys. Acta Mater. 59, 2243-2267 (2011).

4. Greer, A. L. Metallic glasses, Chapter 4 in Physical Metallurgy, $5^{\text {th }}$ edition (eds Laughlin, D. E. \& Hono, K.), Vol. 1, 305-385 (Elsevier, Oxford, 2014). 
5. Wang, W. H. Bulk metallic glasses with functional physical properties. $A d v$. Mater. 21, 4524-4544 (2009).

6. Inoue, A. \& Takeuchi, A. Recent development and application products of bulk glassy alloys. Acta Mater. 59, 2243-2267 (2011).

7. Inoue, A. et al. Development and applications of Fe- and Co-based bulk glassy alloys and their prospects. J. Alloys Comp. 615, S2-S8 (2014).

8. Liebermann, H. H. The dependence of the geometry of glassy alloy ribbons on the chill block melt-spinning process parameters. Mater. Sci. Eng. 43, 203-210 (1980).

9. Ashby, M. F. \& Greer, A. L. Metallic glasses as structural materials. Scripta Mater. 54, 321-326 (2006).

10. $\mathrm{Xu}$, J. \& Ma, E. Damage-tolerant $\mathrm{Zr}-\mathrm{Cu}-\mathrm{Al}$-based bulk metallic glasses with record-breaking fracture toughness. J. Mater. Res. 29, 1489-1499 (2014).

11. Greer, A. L. New horizons for glass formation and stability. Nature Mater. 14, 542-546 (2015).

12. Ren, S., Chen, D. and Zhao, X. Effect of ceramic rolling on the mechanical properties of $\mathrm{Ti}_{42.5} \mathrm{Cu}_{42.5} \mathrm{Ni}_{10} \mathrm{Zr}_{5}$ bulk metallic glass composite. Mater. Sci. Eng. A 646, 90-95 (2015).

13. Verlinden, B., Driver, J., Samajdar, I. \& Doherty, R. D. Thermo-Mechanical Processing of Metallic Materials (Elsevier, Amsterdam, 2007).

14. Wang, W. H. The elastic properties, elastic models and elastic perspectives of metallic glasses. Prog. Mater. Sci. 57, 487-656 (2012).

15. Weaire, D., Ashby, M. F., Logan, J. \& Weins, M. J. On the use of pair potentials to calculate the properties of amorphous metals. Acta Metall. 19, 779-788 (1971).

16. Wang, G., Mattern, N., Pauly, S., Bednarčik, J. \& Eckert, J. Atomic structure evolution in bulk metallic glass under compressive stress. Appl. Phys. Lett. 95, 251906 (2009).

17. Egami, T., Iwashita, T. \& Dmowski, W. Mechanical properties of metallic glasses. Metals 3, 77-113, (2013).

A comprehensive review of the fundamentals of elastic and plastic deformation in metallic glasses.

18. Egami, T. Atomic level stresses. Prog. Mater. Sci. 56, 637-653 (2011).

19. Kuršumović, A. \& Cantor, B. Anelastic crossover and creep recovery spectra in $\mathrm{Fe}_{40} \mathrm{Ni}_{40} \mathrm{~B}_{20}$ metallic glass. Scripta Mater. 34, 1655-1660 (1996).

20. Dmowski, W., Iwashita, T., Chuang, A., Almer, J. \& Egami, T. Elastic heterogeneity in metallic glasses. Phys. Rev. Lett. 105, 205502 (2010).

21. Sun, Y. H., Louzguine-Luzgin, D. V., Ketov, S. \& Greer, A. L. Pure shear stress reversal on a Cu-based bulk metallic glass reveals a Bauschinger-type effect. $J$. Alloys Comp. 615, S75-S78 (2014).

22. Spaepen, F. A microscopic mechanism for steady state inhomogeneous flow in metallic glasses. Acta Metall. 25, 407-415 (1977). 
A fundamental study of deformation mechanisms in metallic glasses, with an analysis based on free volume as the order parameter.

23. Greer, A. L., Cheng, Y. Q. \& Ma, E. Shear bands in metallic glasses. Mater. Sci. Eng. $R$ 74, 71-132 (2013).

A comprehensive review of shear bands and an explanation of the concept of shear-band engineering to improve mechanical properties.

24. Johnson, W. L. \& Samwer, K. A universal criterion for plastic yielding of metallic glasses with a $\left(T / T_{\mathrm{g}}\right)^{2 / 3}$ temperature dependence. Phys. Rev. Lett. 95, 195501 (2005).

25. Argon, A. S. Plastic deformation in metallic glasses. Acta Metall. 27, 47-58 (1979).

A pioneering study that established the concept of the shear transformation zone as the flow unit in metallic glasses.

26. Schall, P., Weitz, D. A. \& Spaepen, F. Structural rearrangements that govern flow in colloidal glasses. Science 318, 1895-1899 (2007).

27. Falk, M. L. \& Langer, J. S. Dynamics of viscoplastic deformation in amorphous solids. Phys. Rev. E 57, 7192-7205 (1998).

28. Takeuchi, S. \& Edagawa, K. Atomistic simulation and modeling of localized shear deformation in metallic glasses. Prog. Mater. Sci. 56, 785-816 (2011).

29. Falk, M. L. \& Langer J. S. Deformation and failure of amorphous, solidlike materials. Ann. Rev. Cond. Matt. Phys. 2, 353-373 (2011).

30. Shang, B. S., Li, M. Z., Yao, Y. G., Lu, Y. J. \& Wang, W. H. Evolution of atomic rearrangements in deformation in metallic glasses. Phys. Rev. E 90, 042303 (2014).

31. Krisponeit, J.-O., Pitikaris, S., Avila, K. E., Küchemann, S., Krüger, A. \& Samwer, K. Crossover from random three-dimensional avalanches to correlated nano shear bands in metallic glasses. Nat. Commun. 5, 3616 (2014).

32. Tao, P.-J. et al. Zr-based bulk metallic glass with super-plasticity under uniaxial compression at room temperature. J. Non-Cryst. Solids 354, 3742-3746 (2008).

33. Hebert, R. J. \& Perepezko, J. H. Effect of cold-rolling on the crystallization behaviour of amorphous $\mathrm{Al}_{88} \mathrm{Y}_{7} \mathrm{Fe}_{5}$ alloy. Mater. Sci. Eng. A 375-377, 728-732 (2004).

34. Cao, Q. P. et al. Effect of pre-existing shear bands on the tensile mechanical properties of a bulk metallic glass. Acta Mater. 58, 1276-1292 (2010).

35. Takayama, S. Drawing of $\mathrm{Pd}_{77.5} \mathrm{Cu}_{6} \mathrm{Si}_{16.5}$ metallic glass wires. Mater. Sci. Eng. 38, 41-48 (1979).

36. Meng, F., Tsuchiya, K., II, S. \& Yokoyama, Y. Reversible transition of deformation mode by structural rejuvenation and relaxation in bulk metallic glass. Appl. Phys. Lett. 101, 121914 (2012).

The high-pressure torsion applied to a $\mathrm{Zr}$-based metallic glass has exerted the largest plastic strain and induced the highest stored energy of any study so far. 
37. Cao, Y. et al. Laser shock peening on Zr-based bulk metallic glass and its effect on plasticity: experiment and modeling. Sci. Rep. 5, 10789 (2015).

38. Concustell, A., Méar, F. O., Suriñach, S., Baró, M. D. \& Greer, A. L. Structural relaxation and rejuvenation in a metallic glass induced by shot-peening. Philos. Mag. Lett. 89, 831-840 (2009).

39. Yavari, A. R. et al. Excess free volume in metallic glasses measured by X-ray diffraction. Acta Mater. 53, 1611-1619 (2005).

40. Jiang, W. H., Pinkerton, F. E. \& Atzmon, M. Mechanical behavior of shear bands and the effect of their relaxation in a rolled amorphous Al-based alloy. Acta Mater. 53, 3469-3477 (2005).

41. He, L. et al. Orientation effect of pre-introduced shear bands in a bulk-metallic glass on its "work-ductilising". Mater. Sci. Eng. A 496, 285-290 (2008).

42. Liu, J. W., Cao, Q. P., Chen, L. Y., Wang, X. D. \& Jiang, J. Z. Shear band evolution and hardness change in cold-rolled bulk metallic glasses. Acta Mater. 58, 4827-4840 (2010).

43. Song, K. K. et al. Significant tensile ductility induced by cold rolling in $\mathrm{Cu}_{47.5} \mathrm{Zr}_{47.5} \mathrm{Al}_{5}$ bulk metallic glass, Intermetall. 19, 1394-1398 (2011).

44. Scudino, S., Jerliu, B., Surreddi, K. B., Kühn, U. \& Eckert, J. Effect of cold rolling on compressive and tensile mechanical properties of $\mathrm{Zr}_{52.5} \mathrm{Ti}_{5} \mathrm{Cu}_{18} \mathrm{Ni}_{14.5} \mathrm{Al}_{10}$ bulk metallic glass. J. Alloys Comp. 509, S128-S130 (2011).

45. Yokoyama, Y., Yamano, K., Fukaura, K., Sunada, H. \& Inoue, A. Ductility improvement of $\mathrm{Zr}_{55} \mathrm{Cu}_{30} \mathrm{Al}_{10} \mathrm{Ni}_{5}$ bulk amorphous alloy. Scripta Mater. 44, 1529-1534 (2001).

46. Lee, M. H. et al. Deformation-induced microstructural heterogeneity in monolithic $\mathrm{Zr}_{44} \mathrm{Ti}_{11} \mathrm{Cu}_{9.8} \mathrm{Ni}_{10.2} \mathrm{Be}_{25}$ bulk metallic glass. Phys. Stat. Solidi RRL 3, 46-48 (2009).

47. Zhang, Y., Wang, W. H. \& Greer, A. L. Making metallic glasses plastic by control of residual stress. Nature Mater. 5, 857-860 (2006).

48. Scudino, S. et al. Ductile bulk metallic glasses produced through designed heterogeneities. Scripta Mater. 65, 815-818 (2011).

49. Liu, Y., Schumacher, G., Riesemeier, H. \& Banhart, J. Change in atomic coordination in a heavily deformed metallic glass. J. Appl. Phys. 115, 203510 (2014).

50. Waseda, Y., Aust, K. T. \& Masumoto, T. Structural changes in amorphous $\mathrm{Pd}_{77} \mathrm{Si}_{17} \mathrm{Cu}_{6}$ due to cold rolling and low temperature annealing. Scripta Metall. 13, 187-190 (1979).

51. Haruyama, O. et al. Characterization of free volume in cold-rolled $\mathrm{Zr}_{55} \mathrm{Cu}_{30} \mathrm{Ni}_{5} \mathrm{Al}_{10}$ bulk metallic glasses. Acta Mater. 61, 3224-3232 (2013).

52. Vempati, U. K., Valavala, P. K., Falk. M. L., Almer, J. \& Hufnagel, T. C. Length-scale dependence of elastic strain from scattering measurements in metallic glasses. Phys. Rev. B 85, 214201 (2012). 
53. Bever, M. B., Holt, D. L. \& Titchener, A. L. The stored energy of cold work. Prog. Mater. Sci. 17, 5-177 (1972).

A comprehensive review of the stored energy in the plastic deformation of crystalline alloys.

54. Hasan, O. A. \& Boyce, M. C. Energy storage during inelastic deformation of glassy polymers. Polym. 34, 5085-5092 (1993).

55. Chen, H. S. Stored energy in a cold-rolled metallic glass. Appl. Phys. Lett. 29, 328-330 (1976).

An early study on the stored energy of cold work in a metallic glass.

56. Fecht, H. J., Hellstern, E., Fu, Z. \& Johnson, W. L. Nanocrystalline metals prepared by high-energy ball milling. Metall. Trans. A 21A, 2333-2337 (1990).

57. Mehrtens, A., von Minnigerode, G., Oelgeschläger, D. \& Samwer, K. Amorphization of the intermetallic compounds $\mathrm{Co}_{2} \mathrm{Zr}$ and $\mathrm{Fe}_{2} \mathrm{Zr}$ under mechanical grinding. Z. Phys. B 88, 25-34 (1992).

58. Grant, D. M., Green, S. M. \& Wood. J. V. The surface performance of shot peened and ion implanted NiTi shape memory alloy. Acta Metall. Mater. 43, 1045-1051 (1995).

59. Busch, R., Schroers, J. \& Wang, W. H. Thermodynamics and kinetics of bulk metallic glass. MRS Bull. 32, 620-623 (2007).

60. Battezzati, L., Riontino, G., Baricco, M., Lucci, A. \& Marino, F. A DSC study of structural relaxation in metallic glasses prepared with different quenching rates. J. Non-Cryst. Solids 61-62, 877-882 (1984).

61. Jessen, B. \& Woldt, E. Stored energy of the deformed metallic glass $\mathrm{Ni}_{78} \mathrm{Si}_{8} \mathrm{~B}_{14}$, Thermochim. Acta 151, 179-186 (1989).

62. Bokeloh, J., Divinski, S. V., Reglitz, G. \& Wilde, G. Tracer measurements of atomic diffusion inside shear bands of a bulk metallic glass. Phys. Rev. Lett. 107, 235503 (2011).

63. Jiang, W. H., Pinkerton, F. E. \& Atzmon, M. Deformation-induced nanocrystallization: A comparison of two amorphous Al-based alloys. J. Mater. Res. 20, 696-702 (2005).

64. Pan, J., Chen, Q., Liu, L. \& Li, Y. Softening and dilatation in a single shear band. Acta Mater. 59, 5146-5158 (2011).

65. Maaß, R., Samwer, K., Arnold, K. \& Volkert, C. A. A single shear band in a metallic glass: Local core and wide soft zone. Appl. Phys. Lett. 105, 171902 (2014).

66. Méar, F. O., Lenk, B., Zhang, Y. \& Greer, A. L. Structural relaxation in a heavily cold-worked metallic glass. Scripta Mater. 59, 1243-1246 (2008).

67. González, S. et al. Influence of the shot-peening intensity on the structure and near-surface mechanical properties of $\mathrm{Ti}_{40} \mathrm{Zr}_{10} \mathrm{Cu}_{38} \mathrm{Pd}_{12}$ bulk metallic glass. Appl. Phys. Lett. 103, 211907 (2013).

68. Méar, F. O., Doisneau, B., Yavari, A. R. \& Greer, A. L. Structural effects of shot-peening in bulk metallic glasses. J. Alloys Comp. 483, 256-259 (2009). 
69. Schroers, J. et al. Thermoplastic blow molding of metals. Mater. Today 14, 1419 (2011).

70. Kumar, G., Tang, H. X. \& Schroers, J. Nanomoulding with amorphous metals. Nature 457, 868-872 (2009).

71. Johnson, W. L. et al. Beating crystallization in glass-forming metals by millisecond heating and processing. Science 332, 828-833 (2011).

72. Way, C., Wadhwa, P. \& Busch, R. The influence of shear rate and temperature on the viscosity and fragility of the $\mathrm{Zr}_{41.2} \mathrm{Ti}_{13.8} \mathrm{Cu}_{12.5} \mathrm{Ni}_{10.0} \mathrm{Be}_{22.5}$ metallic-glassforming liquid. Acta Mater. 55, 2977-2983 (2007).

73. Demetriou, M. D. \& Johnson, W. L. Shear flow characteristics and crystallization kinetics during steady non-isothermal flow of Vitreloy-1. Acta Mater. 52, 3403-3412 (2004).

74. Shao, Z. et al. Shear-accelerated crystallization in a supercooled atomic liquid. Phys. Rev. E 91, 020301(R) (2015).

75. Tong, Y. et al. Recovering compressive plasticity of bulk metallic glasses by high-temperature creep. Scripta Mater. 69, 570-573 (2013).

Shows that creep can induce rejuvenation in metallic glasses and can restore plasticity to samples embrittled by annealing.

76. Tong, Y. et al. Structural rejuvenation in bulk metallic glasses. Acta Mater. 86, 240-246 (2015).

77. Ichitsubo, T., Matsubara, E., Yamamoto, T., Chen, H. S., Nishiyama, N., Saida, J. \& Anazawa, K. Microstructure of fragile metallic glasses inferred from ultrasound-accelerated crystallization in Pd-based metallic glasses. Phys. Rev. Lett. 95, 245501 (2005).

78. Wang, Y., Zhao, W., Li, G. \& Liu, R. Effects of ultrasonic treatment on the structure and properties of Zr-based bulk metallic glasses. J. Alloys Comp. 544, 46-49 (2012).

79. Packard, C. E., Franke, O., Homer, E. R. \& Schuh, C. A. Nanoscale strength distribution in amorphous versus crystalline metals. J. Mater. Res. 25, 22512263 (2010).

80. Packard, C. E., Homer, E. R., Al-Aqeeli, N. \& Schuh, C. A. Cyclic hardening of metallic glasses under Hertzian contacts: Experiments and STZ dynamics simulations. Philos. Mag. 90, 1373-1390 (2010).

A demonstration that cyclic loading in the elastic range can induce a hardening effect in metallic glasses.

81. Al-Aqeeli, N. Strengthening behaviour due to cyclic elastic loading in Pd-based metallic glass. J. Alloys Comp. 509, 7216-7220 (2011).

82. Deng, C. \& Schuh, C. A. Atomistic mechanisms of cyclic hardening in metallic glass. Appl. Phys. Lett. 100, 251909 (2012).

83. Cao, R., Deng, Y. \& Deng, C. Hardening and crystallization in monatomic metallic glass during elastic cycling. J. Mater. Res. 30, 1820-1826 (2015).

84. El-Shabasy, A. B. \& Lewandowski, J. J. Fatigue coaxing experiments on a Zrbased bulk-metallic glass. Scripta Mater. 62, 481-484 (2010). 
85. Tong, P. et al. Structural irreversibility and enhanced brittleness under fatigue in Zr-based amorphous solids. Metals 2, 529-539 (2012).

86. Ye, J. C., Lu, J., Liu, C. T., Wang, Q. \& Yang, Y. Atomistic free-volume zones and inelastic deformation of metallic glasses. Nature Mater. 9, 619-623 (2010). A demonstration of hysteretic behaviour on cycling in the elastic range.

87. Wang, Z., Wen, P., Huo, L. S., Bai, H. Y. \& Wang, W. H. Signature of viscous flow units in apparent elastic regime of metallic glasses. Appl. Phys. Lett. 101, 121906 (2012).

88. Caron, A., Kawashima, A., Fecht, H.-J., Louzguine-Luzguin, D. V. \& Inoue, A. On the anelasticity and strain induced structural changes in a Zr-based bulk metallic glass. Appl. Phys. Lett. 99, 171907 (2011).

89. Yu, H.-B., Wang, W.-H., Samwer, K. The $\beta$ relaxation in metallic glasses: an overview. Mater. Today 16, 183-191 (2013).

90. Tian, L. et al. Approaching the ideal elastic limit of metallic glasses. Nat. Commun. 3, 609 (2012).

91. Wang, Z. T., Pan, J., Li, Y. \& Schuh, C. A. Densification and strain hardening of a metallic glass under tension at room temperature. Phys. Rev. Lett. 111, 135504 (2013)

92. Wang, Y., Zhao, W., Li, G., Li, Y. \&Liu, R. Structural evolution of lanthanidebased metallic glasses under high pressure annealing. J. Alloys Comp. 551, 185188 (2013).

93. Lee, S.-C., Lee, C.-M., Yang, J.-W. \& Lee, J.-C. Microstructural evolution of an elastostatically compressed amorphous alloy and its influence on the mechanical properties. Scripta Mater. 58, 591-594 (2008).

94. Park, K. W. et al. Elastostatically induced structural disordering in amorphous alloys. Acta Mater. 56, 5440-5454 (2008).

95. Ke, H. B., Wen, P., Peng, H. L., Wang, W. H. \& Greer, A. L. Homogeneous deformation of metallic glass at room temperature reveals large dilatation. Scripta Mater. 64, 966-969 (2011).

96. Lee, J.-C. Calorimetric study of $\beta$-relaxation in an amorphous alloy: an experimental technique for measuring the activation energy for shear transformation. Intermetall. 44, 116-120 (2014).

A study on rejuvenation of metallic glasses by static loading in the elastic range.

97. Greer, A. L. \& Sun, Y. H. Stored energy in metallic glasses due to strains within the elastic limit. Philos. Mag. 96, (2016) in press, DOI:10.1080/14786435.2016.1177231.

98. Gu, J., Song, M., Ni, S., Liao, X. \& Guo, S. Improving the plasticity of bulk metallic glasses via pre-compression below the yield stress. Mater. Sci. Eng. A 602, 68-76 (2014).

99. Lee, C.-M., Park, K.-W. \& Lee, J.-C. Plasticity improvement of a bulk amorphous alloy based on its viscoelastic nature. Scripta Mater. 59, 802-805 (2008). 
100. Ketov, S. V. et al. Rejuvenation of metallic glasses by non-affine thermal strain. Nature 524, 200-203 (2015).

This study shows that thermal cycling well below the glass-transition temperature can induce rejuvenation and can restore plasticity to samples embrittled by annealing.

101. Lacks, D. J. \& Osborne, M. J. Energy landscape picture of overaging and rejuvenation in a sheared glass. Phys. Rev. Lett. 93, 255501 (2004).

102. Fiocco, D., Foffi, G., \& Sastry, S. Oscillatory athermal quasistatic deformation of a model glass. Phys. Rev. E 88, 020301 (2013).

103. Fiocco, D., Foffi, G. \& Sastry, S. Encoding of memory in sheared amorphous solids. Phys. Rev. Lett. 112, 025702 (2014).

104. Egami, T., Flanders, P. J. \& Graham Jr., C. D. Low-field magnetic properties of ferromagnetic amorphous alloys. Appl. Phys. Lett. 26, 128-130 (1975).

105. Spilsbury, D., Butvin, P., Cowlam, N., Howells, W. S. \& Cooper, R. J. Some evidence for 'directional atomic pair ordering' in a cobalt-based metallic glass. Mater. Sci. Eng. A 226-228, (1997) 187-191 (1997).

106. Tarumi, R. et al. Elastic anisotropy of an $\mathrm{Fe}_{79} \mathrm{Si}_{12} \mathrm{~B}_{9}$ amorphous alloy thin film studied by ultrasound spectroscopy. J. Appl. Phys. 101, 053519 (2007).

107. Berry, B. S. \& Pritchet, W. C. Magnetic annealing and directional ordering of an amorphous ferromagnetic alloy, Phys. Rev. Lett. 34, 1022-1025 (1975).

108. González, J., Vázquez, M., Barandiarán, J. M., Madurga, V. \& Hernando, A. Different kinds of magnetic anisotropies induced by current annealing in metallic glasses. J. Magn. Magn. Mater. 68, 151-156 (1987).

109. Arakawa, S. et al. Anisotropy of tensile properties of amorphous metals, in Rapidly Quenched Metals (eds Steeb, S. \& Warlimont, H.) 1389-1392 (NorthHolland, Amsterdam, 1985).

110. Concustell, A., Godard-Desmarest, S., Carpenter, M. A., Nishiyama, N. \& Greer, A. L. Induced elastic anisotropy in a bulk metallic glass. Scripta Mater. 64, 1091-1094 (2011).

A study that shows frozen-in anelastic strain and viscous flow lead to anisotropy of opposite sign.

111. Sun, Y. H., Concustell, A., Carpenter, M. A., Qiao, J. C., Rayment, A. W. \& Greer, A. L. Flow-induced elastic anisotropy of metallic glasses. Acta Mater. 112, 132-140 (2016).

112. Ott, R. T. et al. Anelastic strain and structural anisotropy in homogeneously deformed $\mathrm{Cu}_{64.5} \mathrm{Zr}_{35.5}$ metallic glass. Acta Mater. 56, 5575-5583 (2008).

113. Dmowski, W. \& Egami, T. Structural anisotropy in metallic glasses induced by mechanical deformation. Adv. Eng. Mater. 10, 1003-1007 (2008).

A structural study of induced anisotropy.

114. Ott, R. T., Kramer, M. J., Besser, M. F. \& Sordelet, D. J. High-energy X-ray measurements of structural anisotropy and excess free volume in a homogenously deformed Zr-based metallic glass. Acta Mater. 54, 2463-2471 (2006). 
115. Nielsen, O. V. \& Nielsen, H. J. V. Magnetic anisotropy in $\mathrm{Co}_{73} \mathrm{Mo}_{2} \mathrm{Si}_{15} \mathrm{~B}_{10}$ and $\left(\mathrm{Co}_{0.89} \mathrm{Fe}_{0.11}\right)_{72} \mathrm{Mo}_{3} \mathrm{Si}_{15} \mathrm{~B}_{10}$ metallic glasses, induced by stress-annealing, $J$. Magn. Magn. Mater. 22, 21-24 (1980).

116. Nielsen, O. V., Hernando, A., Madurga, V. \& Gonzalez, J. M. Experiments concerning the origin of stress anneal induced magnetic anisotropy in metallic glass ribbons, J. Magn. Magn. Mater. 46, 341-349 (1985).

117. Suzuki, Y., Haimovich, J. \& Egami T. Bond-orientational anisotropy in metallic glasses observed by x-ray diffraction. Phys. Rev. B 35, 2162-2168 (1987).

118. Baumer, R. E. \& Demkowicz, M. J. Radiation response of amorphous metal alloys: Subcascades, thermal spikes and super-quenched zones. Acta Mater. 83, 419-430 (2015).

119. Mayr, S. G. Impact of ion irradiation on the thermal, structural, and mechanical properties of metallic glasses. Phys. Rev. B 71, 144109 (2005).

120. Zhang, H., Mei, X., Wang, Y., Wang, Z. \& Wang, Y. Resistance to $\mathrm{H}^{+}$induced irradiation damage in metallic glass $\mathrm{Fe}_{80} \mathrm{Si}_{7.43} \mathrm{~B}_{12.57}$. J. Nucl. Mater. 456, 344350 (2015).

121. Xiao, Q., Huang, L. \& Shi, Y. Suppression of shear banding in amorphous ZrCuAl nanopillars by irradiation. J. Appl. Phys. 113, 083514 (2013).

122. Huang, Y. et al. Structure and mechanical property modification of a Ti-based metallic glass by ion irradiation. Scripta Mater. 103, 41-44 (2015).

123. Carter, J. et al. Effects of ion irradiation in metallic glasses. Nucl. Instrum. Meth. Phys. Res. B 267, 1518-1521 (2009).

124. Nagase, T. et al. $\mathrm{MeV}$ electron irradiation induced crystallization in metallic glasses: Atomic structure, crystallization mechanism and stability of an amorphous phase under the radiation. J. Non-Cryst. Solids 358, 502-518 (2012).

125. Miglierini, M. \& Hasiak, M. Impact of ion irradiation upon structure and magnetic properties of NANOPERM-type amorphous and nanocrystalline alloys. J. Nanomater. 2015, 175407 (2015).

126. Kramer, E. A., Johnson, W. L. \& Cline, C. The effects of neutron irradiation on a superconducting metallic glass. Appl. Phys. Lett. 35, 815-818 (1979).

127. Raghavan, R., Boopathy, K., Ghisleni, R., Pouchon, M. A., Ramamurty, U. \& Michler, J. Ion irradiation enhances the mechanical performance of metallic glasses. Scripta Mater. 62, 462-465 (2010).

128. Avchaciov, K. A., Ritter, Y., Djurabekova, F., Nordlund, K. \& Albe, K. Effect of ion irradiation on structural properties of $\mathrm{Cu}_{64} \mathrm{Zr}_{36}$ metallic glass. Nucl. Instrum. Meth. Phys. Res. B 341, 22-26 (2014).

129. Gerling, R., Schimansky, F. P. \& Wagner, R. Restoration of the ductility of thermally embrittled amorphous alloys under neutron-irradiation. Acta Metall. 35, 1001-1006 (1987).

An important study showing that neutron irradiation can repeatedly restore plasticity to metallic glasses embrittled by thermal annealing. The damage level (displacements per atom) required to restore plasticity is characterized. 
130. Ediger, M. D. \& Harrowell, P. Perspective: Supercooled liquids and glasses. $J$. Chem. Phys. 137, 080901 (2012).

131. Swallen, S. F. et al. Organic glasses with exceptional thermodynamic and kinetic stability. Science 315, 353-356 (2007).

132. Yu, H.-B., Luo, Y. \& Samwer, K. Ultrastable metallic glass. Adv. Mater. 25, 5904-5908 (2013).

133. Aji, D. P. B. et al. Ultrastrong and ultrastable metallic glass. arXiv:1306.1575 [cond-mat.mtrl-sci], (2013).

134. Wang, J. Q. et al. The ultrastable kinetic behavior of an Au-based nanoglass. Acta Mater. 79, 30-36 (2014).

135. Gleiter, H. Our thoughts are ours, their ends none of our own: Are there ways to synthesize materials beyond the limitations of today? Acta Mater. 56, 58755893 (2008).

An introduction and overview of the concept of 'nanoglasses'.

136. Fang, J. X. et al. Atomic structure and structural stability of $\mathrm{Sc}_{75} \mathrm{Fe}_{25}$ nanoglasses. Nano Lett. 12, 458-463 (2012).

137. Wang, X. L. et al. Plasticity of a scandium-based nanoglass. Scripta Mater. 98, 40-43 (2015).

138. Franke, O., Leisen, D., Gleiter, H. \& Hahn, H. Thermal and plastic behavior of nanoglasses. J. Mater. Res. 29, 1210-1216 (2014).

139. Wang, X. D. et al. Atomic-level structural modifications induced by severe plastic shear deformation in bulk metallic glasses. Scripta Mater. 64, 81-84 (2011).

140. Chen, N. et al. Formation and properties of Au-based nanograined metallic glasses. Acta Mater. 59, 6433-6440 (2011).

141. Ichitsubo, T., Matsubara, E., Anazawa, K. \& Nishiyama, N. Crystallization accelerated by ultrasound in Pd-based metallic glasses. J. Alloys Comp. 434435, 194-195 (2007).

142. Zhong, L., Wang, J., Sheng, H., Zhang, Z. \& Mao, S. X. Formation of monatomic metallic glasses through ultrafast liquid quenching. Nature 512, 177-180 (2014).

143. Magagnosc, D. J. et al. Effect of ion irradiation on tensile ductility, strength and fictive temperature in metallic glass nanowires. Acta Mater. 74, 165-182 (2014).

144. Gallino, I., Schroers, J. \& Busch, R. Kinetic and thermodynamic studies of the fragility of bulk metallic glass forming liquids. J. Appl. Phys. 108, 063501 (2010).

145. Donohue, A., Spaepen, F., Hoagland, R. G. \& Misra, A. Suppression of the shear band instability during plastic flow of nanometer-scale confined metallic glasses. Appl. Phys. Lett. 91, 241905 (2007).

146. Garner, F. A. Chapter 6: Irradiation performance of cladding and structural steels in liquid metal reactors, in Nuclear Materials (Vol. 10A, Materials Science \& Technology: A Comprehensive Treatment, ed. Frost, B. R. D.), p. 436. $\mathrm{VCH}$, Weinheim (1994). 
147. Lu, J., Ravichandran, G. \& Johnson, W. L. Deformation behavior of the $\mathrm{Zr}_{41.2} \mathrm{Ti}_{13.8} \mathrm{Cu}_{12.5} \mathrm{Ni}_{10} \mathrm{Be}_{22.5}$ bulk metallic glass over a wide range of strain-rates and temperatures. Acta Mater. 51, 3429-3443 (2003).

148. Helgeson, M. E., Reichert, M. D., Hu, Y. T. \& Wagner, N. J. Relating shear banding, structure, and phase behavior in wormlike micellar solutions. Soft Matter 5, 3858-3869 (2009).

149. Suryanarayana, C. Mechanical alloying and milling. Prog. Mater. Sci. 46, 1184 (2001).

150. Tong, Y, Dmowski, W., Witczak, Z., Chuang, C.-P. \& Egami, T. Residual elastic strain induced by equal channel angular pressing on bulk metallic glasses. Acta Mater. 61, 1204-1209 (2013).

\section{Acknowledgements}

Y.H.S. is supported by a China Scholarship Council (CSC) scholarship, and A.L.G. by the Engineering and the Engineering and Physical Sciences Research Council, UK and the World Premier International Research Center Initiative (WPI), MEXT, Japan.

\section{Competing interests statement}

The authors declare no competing financial interests. 


\section{FIGURES}

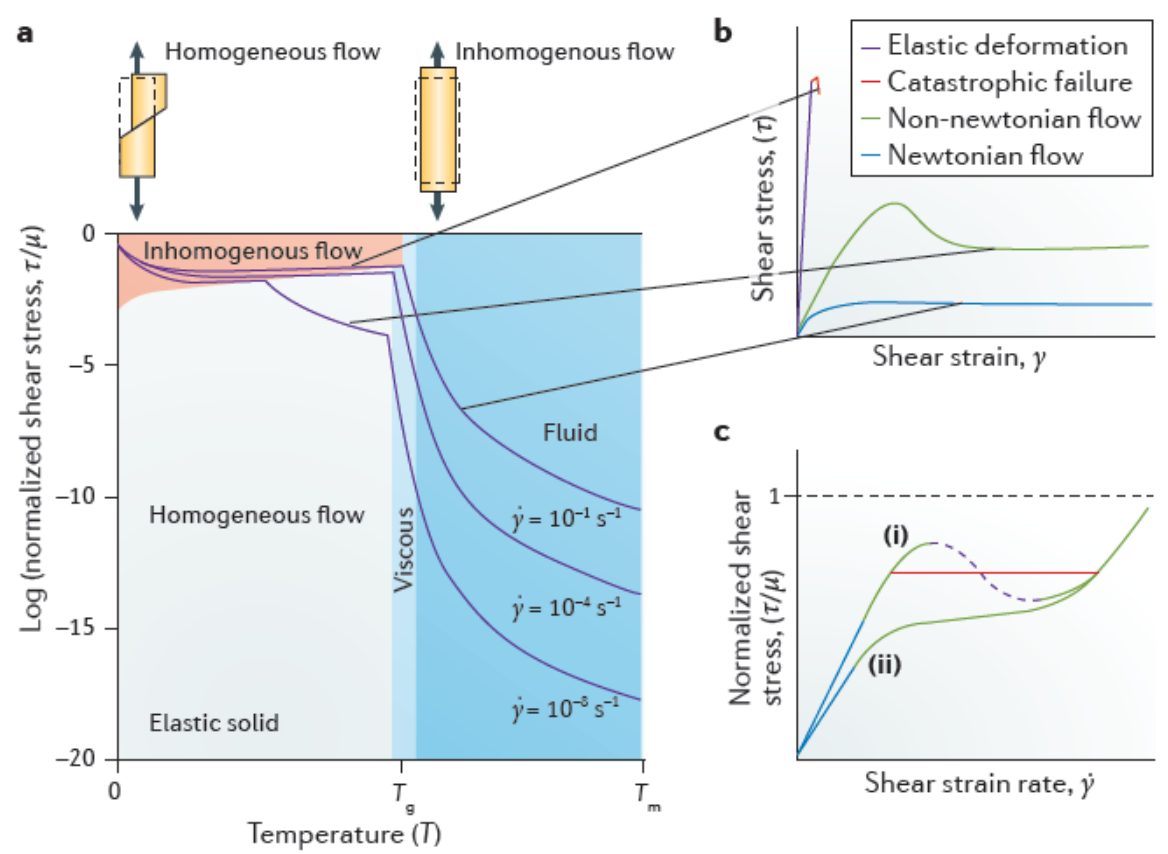

Figure 1 | The deformation of metallic glasses. a| A deformation-mechanism map for a metallic glass and liquid, as suggested by Spaepen ${ }^{22}$. The applied shear stress $\tau$ is normalized by the shear modulus $\mu$. The contours show shear strain rate $\dot{\gamma}$. bl Schematic stress-strain curves for uniaxial compression of a metallic glass at constant temperature, $T$, and $\dot{\gamma}$. Decreasing $T$, or increasing $\dot{\gamma}$, leads to a transition from Newtonian to non-Newtonian flow and ultimately to inhomogeneous flow at the onset of yielding ${ }^{147}$, and therefore to immediate catastrophic failure. cl Sketches of the variation of $\tau$ with $\dot{\gamma}$ at a given temperature. Curve (i) shows the progression from Newtonian (blue line) to non-Newtonian (green) flow at higher $\dot{\gamma}$. The dashed portion of the line represents an unstable flow. Flow can occur according to any of the solid lines, including (red) the inhomogeneous co-existence of two different $\dot{\gamma}$ at the same $\tau$. Shear-banding instabilities of this kind occur in a wide range of systems ${ }^{148}$. Curve (ii) shows possible avoidance of the inhomogeneous-flow instability, realized by operating at higher temperatures or testing a glass that is less relaxed. $T_{\mathrm{g}}$ : glass-transition temperature, $T_{\mathrm{m}}$ : melting temperature. 
a Rolling

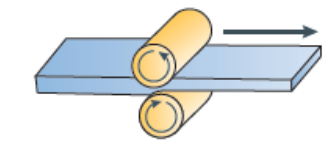

c High-pressure torsion

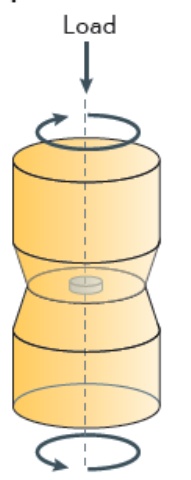

e Ball-milling

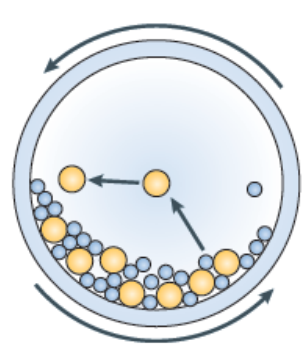

b Wire-drawing

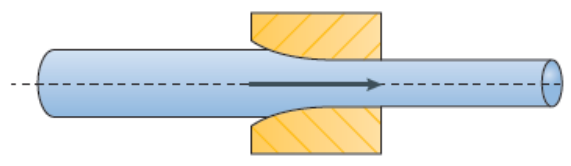

d Shot-peening

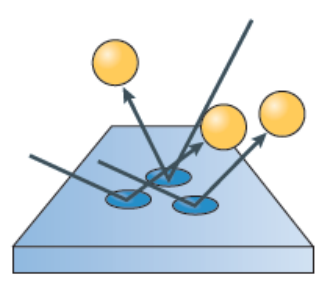

f Equal-channel angular pressing

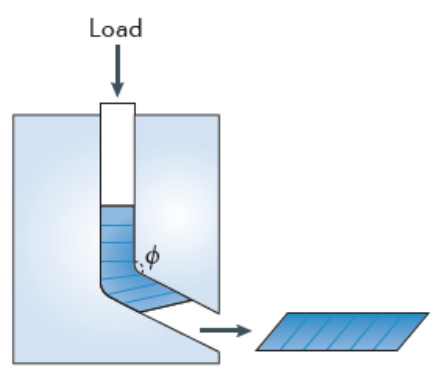

Figure 2 | Cold-working methods that permit heavy plastic deformation of metallic glasses at room temperature. al Applied to samples with initial cross-section ranging from $22 \mu \mathrm{m}$ thick and $1 \mathrm{~mm}$ wide (melt-spun ribbon ${ }^{40}$ ) up to $1.7 \mathrm{~mm}$ thick and typically $10 \mathrm{~mm}$ wide (bulk metallic glass plate ${ }^{34,44}$ ). After multi-stage rolling, thicknesses as low as $12 \mu \mathrm{m}$ have been achieved [40]. b| A cast wire is drawn through a die. Multi-stage drawing can give wires of diameter reduced to $\sim 75 \mu \mathrm{m}$ [REF. 35]. cl The disc-shaped sample (typically $0.85 \mathrm{~mm}$ thick and $10 \mathrm{~mm}$ diameter ${ }^{36}$ ) is subjected to high axial compression and torsion. d/ Repeated impact of beads (typically oxide glass or steel, $0.3-0.7 \mathrm{~mm}$ diameter) deforms the surface to a depth of $\sim 100 \mu \mathrm{m}$ generating a compressive residual stress in the deformed layer ${ }^{38,47,67}$. el Ball-milling has many variants ${ }^{149}$. The sample (usually in powder form) and one or more metallic or ceramic balls (with total mass much greater than the sample) are in a vial that is agitated by rotation or vibration. Repeated impact by the balls can, for example, reduce melt-spun ribbon fragments from an initial thickness of $30 \mu \mathrm{m}$ to $1-$ $3 \mu \mathrm{m}$ [REF. 39]. f| During pressing the cross-section of the sample stays the same; the angle $\phi$ determines whether the shear is elastic or plastic. Sample thickness and width are typically a few $\mathrm{mm}^{99,150}$. 


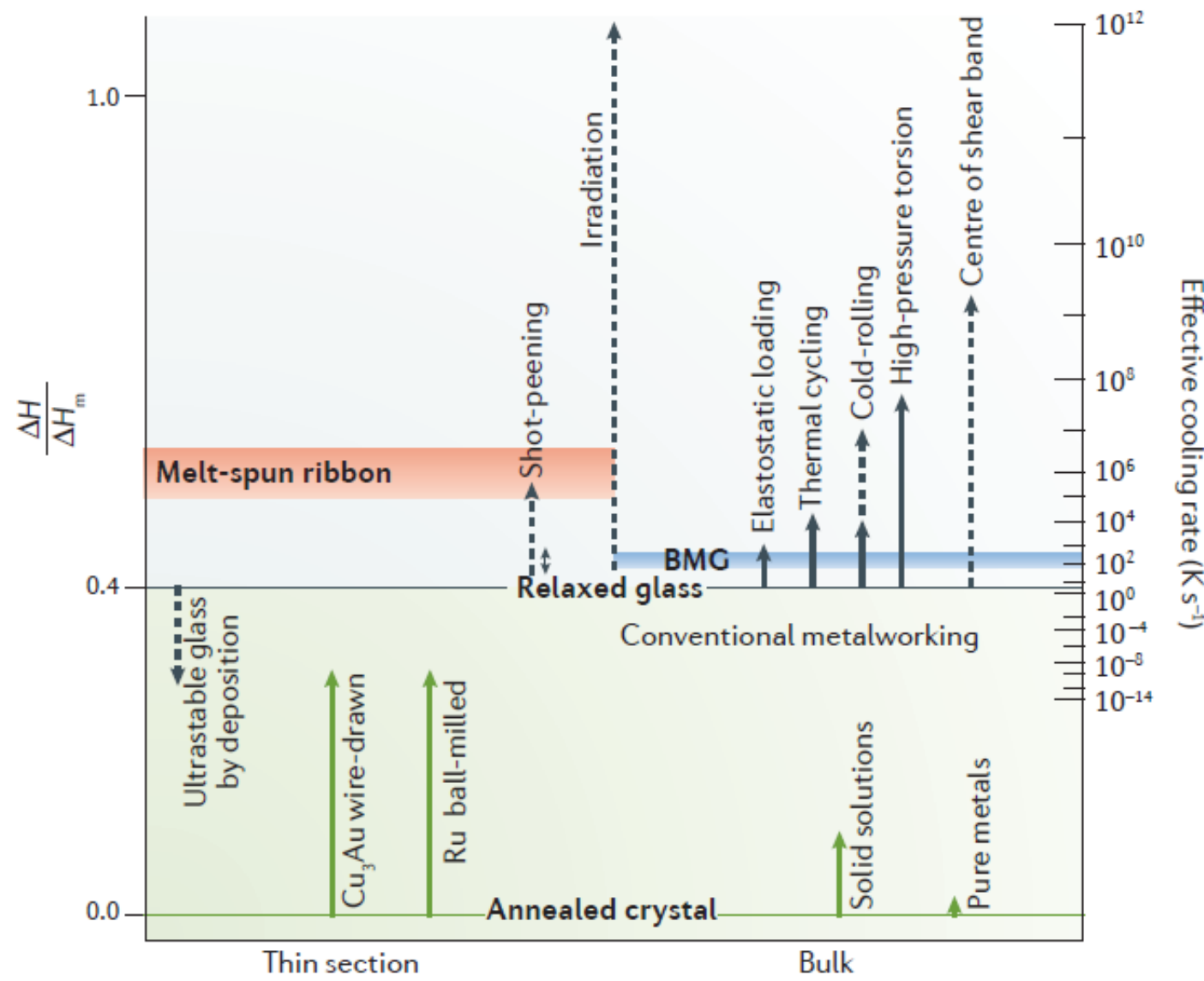

Figure 3 | Relative enthalpies of deformed metallic states at room temperature. The enthalpies are normalized by the heat of melting, with an annealed crystalline state as baseline. Assuming a typical temperature dependence of liquid viscosity ${ }^{143}$, the enthalpy of the glass can be used to estimate the cooling rate at which a glass with that enthalpy would be formed (right-hand axis). The increase in energy stored in metallic glasses as a result of cold-working is similar to that stored in polycrystalline metals; however, in addition to this energy, there is the excess enthalpy represented by the heat of crystallization (the baseline at $0.4 \Delta H / \Delta H_{\mathrm{m}}$ ). Samples available only in thin sections (particles, wires, melt-spun ribbons and thin films), and processes applicable only to thin sections (thin-film deposition, surface treatments, deformation of particle and wires), are shown on the left; bulk samples, and processes applicable to the bulk, on the right. The maximum reported effects of each type of processing are shown by the arrows (green for polycrystals; black for metallic glasses; dashed arrows indicate estimates and solid ones direct measurements). Two initial states of metallic glasses are shown: melt-spun ribbon and bulk metallic glass (BMG). For ultrastable glass, the arrow shows the enthalpy a metallic glass would have if it could be equilibrated at 0.85 of the conventional glasstransition temperature, as for organic glasses ${ }^{130}$. Shot-peening can both lower and 
raise the enthalpy of BMG surface layers ${ }^{38,67}$. Elastostatic loading ${ }^{96}$ and thermal cycling $^{100}$ raise the enthalpy. Cold-rolling results in higher-energy states when applied to melt-spun metallic glasses ${ }^{61}$ (dashed arrow) than when applied to BMGs ${ }^{42}$ (solid arrow). The estimated enthalpy at the centre of a shear band ${ }^{64}$ may represent the highest value attainable by mechanical processing. The highest measured enthalpy is for a sample subjected to high-pressure torsion ${ }^{36}$. Irradiation is applicable to thin surface layers (electrons and ions) and to bulk (neutrons), inducing states comparable to those achieved by cooling at $>10^{13} \mathrm{~K} \mathrm{~s}^{-1}$ [REF. 118]; such states have high enthalpy, but the value is not readily quantifiable. Pure-metal glasses ${ }^{142}$ obtained by cooling at $\sim 10^{14} \mathrm{~K} \mathrm{~s}^{-1}$ are not shown; the cooling rate does not readily indicate an enthalpy, as the temperature dependence of the liquid viscosity is unknown. $\Delta H$ : excess enthalpy, $\Delta H_{\mathrm{m}}$ : latent heat of melting. 
a

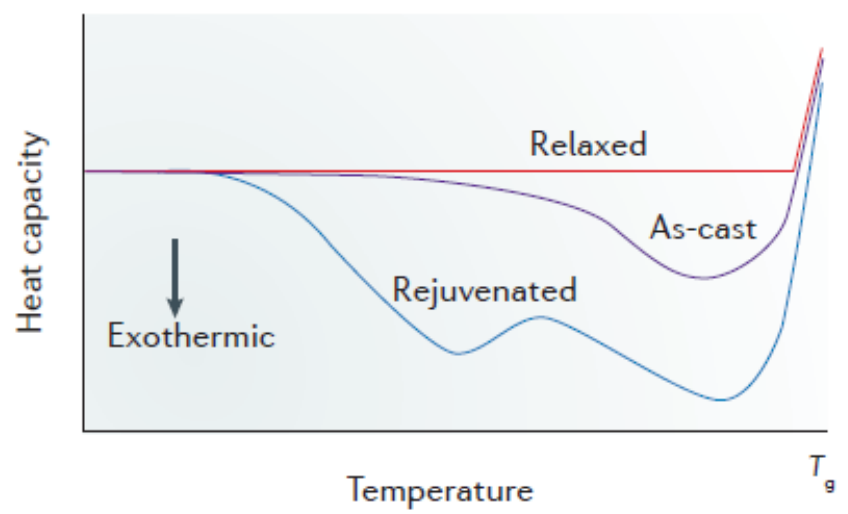

b

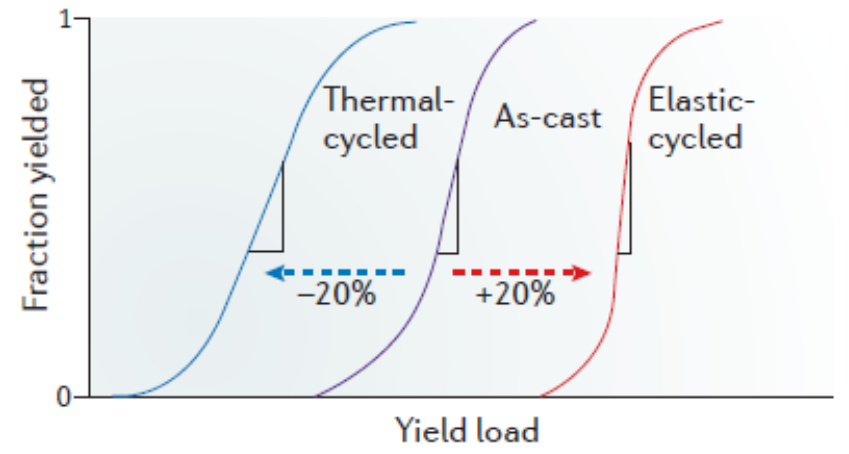

Figure 4 | Relaxation and rejuvenation. al Sketches of differential scanning calorimetry traces of a metallic glass showing an exothermic relaxation spectrum upon heating towards the glass-transition temperature, $T_{\mathrm{g}}$. An as-cast glass shows a heat of relaxation, $\Delta H_{\text {rel, }}$ that is proportional to the area between the purple and red traces; the red trace is obtained for a well annealed glass. After plastic deformation, elastostatic loading or thermal cycling, $\Delta H_{\text {rel }}$ is increased and the relaxation spectrum (blue trace) starts at a lower temperature (as low as $0.55 T_{\mathrm{g}}$ ), often showing a distinct lower-temperature peak that may be related to $\beta$ relaxation ${ }^{89,96,97}$. b| Schematic cumulative distributions of the initial yield load upon nanoindentation of a metallic glass. Prior cyclic loading in the elastic range under the indenter tip shifts the yield loads to higher values, a hardening that can be associated with a more relaxed structure ${ }^{80}$. Thermal cycling ( 11 cycles from room temperature down to $77 \mathrm{~K}$ ) gives an opposite rejuvenation effect ${ }^{100}$ (originally reported in terms of the related yield pressure). Relaxation is associated with a narrower distribution of pop-in loads, implying a more homogeneous glass, whereas the opposite is true for rejuvenation. 
a

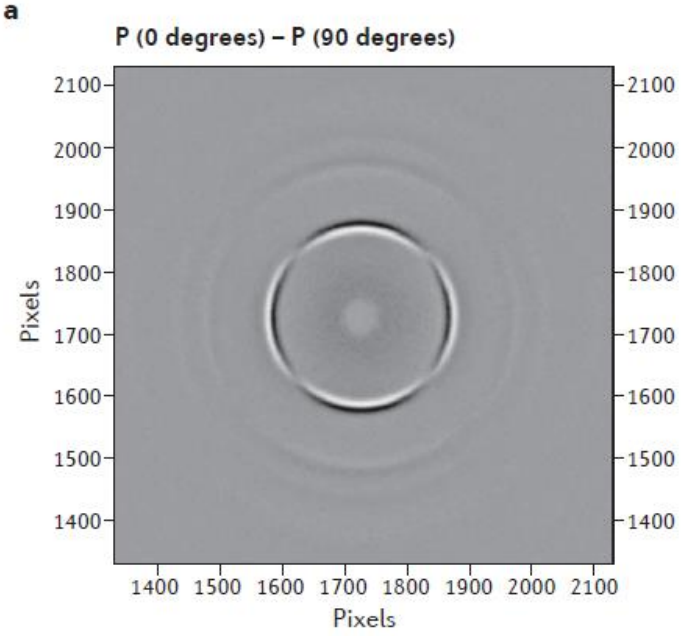

b

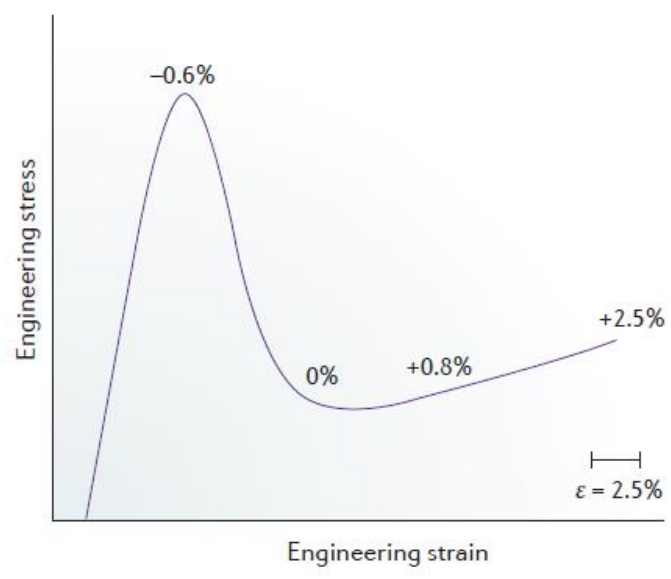

Figure 5 | Anisotropy induced by homogeneous flow. a| The difference between diffraction patterns of an anisotropic $\mathrm{Fe}_{81} \mathrm{~B}_{13} \mathrm{Si}_{14} \mathrm{C}_{2}$ metallic-glass ribbon in two orthogonal orientations indicating that the first halo is elliptical ${ }^{113}$. The ribbon had undergone creep to a strain of $2-3 \%$ by loading in tension at $573 \mathrm{~K}$ for $30 \mathrm{~min}$. The patterns were obtained in transmission with $87 \mathrm{keV} X$-rays. b| The stress-strain curve for a $\mathrm{Pd}_{40} \mathrm{Cu}_{30} \mathrm{Ni}_{10} \mathrm{P}_{20}$ bulk metallic glass under uniaxial compression ${ }^{111}$ (strain rate $10^{-3} \mathrm{~s}^{-1}, 533 \mathrm{~K}$ ). The labels show the percentage elastic anisotropy (defined as $\left(\mu_{4}-\mu_{6}\right) / \mu_{6}$ for shear moduli $\mu_{4}\left(\equiv C_{2323}\right)$ and $\mu_{6}\left(\equiv C_{1212}\right)$ relative to the creep axis $x_{3}$ and transverse plane $x_{1}-x_{2}$ ) measured by resonant ultrasound spectroscopy at room temperature for samples crept to the given strains. Panel al is reprinted with permission from REF. 113, panel b| with permission from REF. 111. 


\section{Box 1 Figure:}

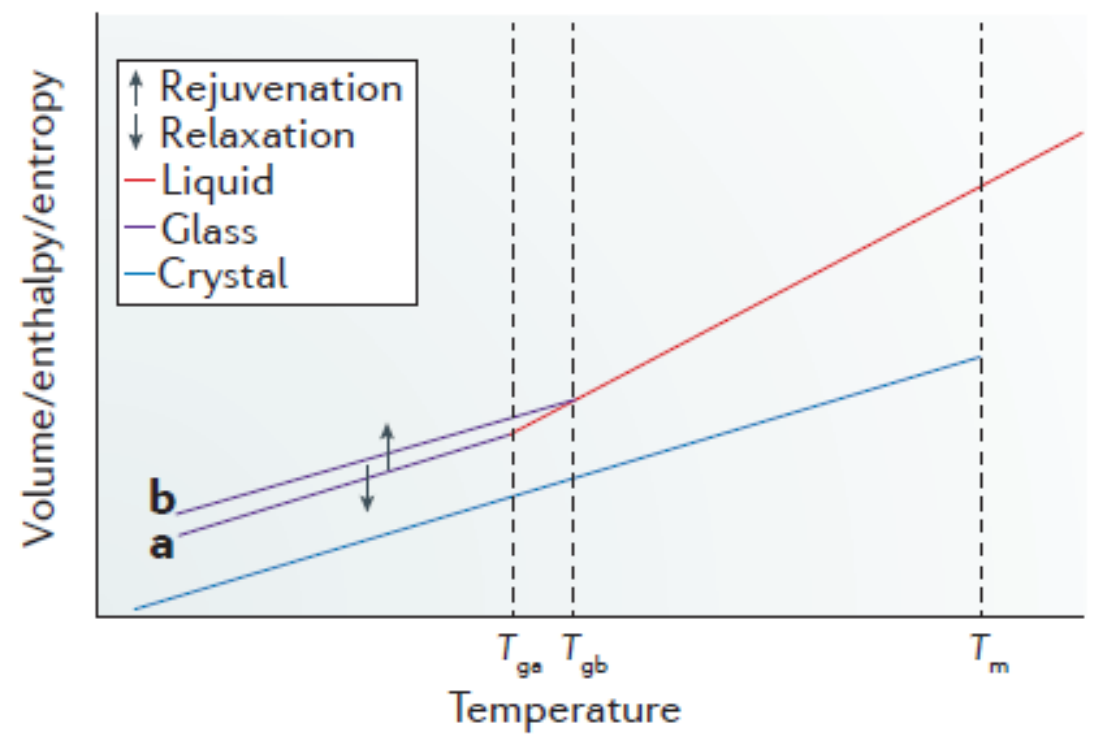

\section{Box 2 Figure:}

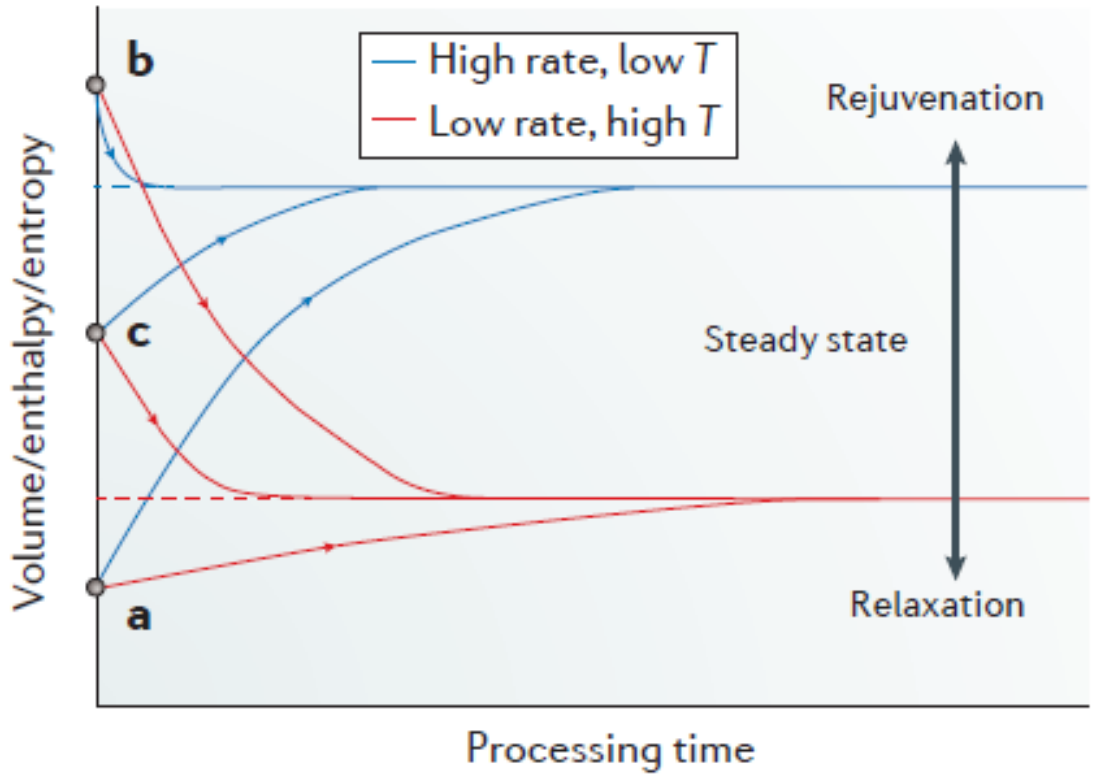

